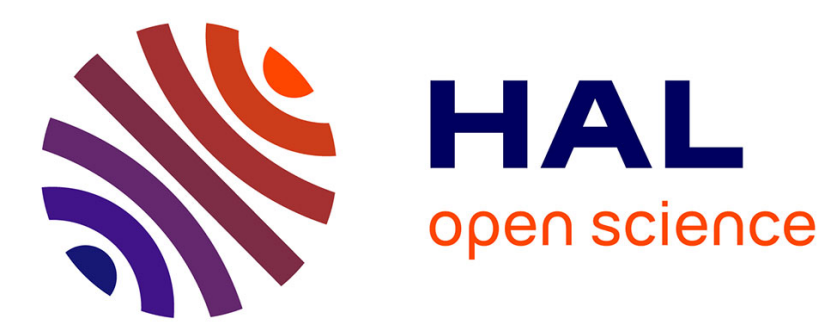

\title{
Periodic motion and bifurcations induced by the Painlevé paradox
}

\author{
Remco Leine, Bernard Brogliato, Henk Nijmeijer
}

\section{To cite this version:}

Remco Leine, Bernard Brogliato, Henk Nijmeijer. Periodic motion and bifurcations induced by the Painlevé paradox. European Journal of Mechanics - A/Solids, 2002, 10.1016/S0997-7538(02)01231-7 . hal-01310673

\section{HAL Id: hal-01310673 \\ https://hal.science/hal-01310673}

Submitted on 3 May 2016

HAL is a multi-disciplinary open access archive for the deposit and dissemination of scientific research documents, whether they are published or not. The documents may come from teaching and research institutions in France or abroad, or from public or private research centers.
L'archive ouverte pluridisciplinaire HAL, est destinée au dépôt et à la diffusion de documents scientifiques de niveau recherche, publiés ou non, émanant des établissements d'enseignement et de recherche français ou étrangers, des laboratoires publics ou privés. 


\title{
Periodic motion and bifurcations induced by the Painlevé paradox
}

\author{
R.I. Leine ${ }^{\mathrm{a}, *}$, B. Brogliato ${ }^{\mathrm{b}}$, H. Nijmeijer ${ }^{\mathrm{a}}$ \\ a Department of Mechanical Engineering, Eindhoven University of Technology, PO Box 513, 5600 MB Eindhoven, The Netherlands \\ b INRIA Rhône-Alpes, ZIRST Montbonnot, 655 avenue de l'Europe, 38334, Saint Ismier cedex, France
}

In this paper we study the periodic motion and bifurcations of the Frictional Impact Oscillator, which consists of an object with normal and tangential degrees of freedom that comes in contact with a rigid surface. The Frictional Impact Oscillator contains the basic mechanism for a hopping phenomenon observed in many practical applications. We will show that the hopping or bouncing motion in this type of systems is closely related to the Painlevé paradox. A dynamical system exhibiting the Painlevé paradox has nonuniqueness and nonexistence of solutions in certain sliding modes. Furthermore, we will show that this type of systems can exhibit the Painlevé paradox for physically realistic values of the friction coefficient.

Keywords: Nonuniqueness; Multibody dynamics; Linear complementarity problem; Contact

\section{Introduction}

If a finger is pushed over a table, then a hopping motion of the finger can be observed when the finger and table are sufficiently rough. The same phenomenon occurs when a piece of chalk is pushed over the blackboard, often resulting in a dotted line which indicates periodic detachment. Brake systems, consisting of a pin-on-disk mechanism, can exhibit an intermittent motion, where the pin periodically loses contact with the disk, accompanied by a squealing noise (Ibrahim, 1994). Robotic manipulators, which touch a surface of an obstacle, may also show a kind of bouncing motion, normally regarded as undesirable (Brogliato, 1999). In this paper we will study a Frictional Impact Oscillator, which consists of a rigid object with normal and tangential degrees of freedom that comes in contact with a rigid surface. The Frictional Impact Oscillator, introduced in this paper, contains the basic mechanism for the hopping phenomenon observed in many practical applications. We will show that the hopping or bouncing motion in this type of systems is closely related to the Painlevé paradox (which we explain in the sequel). Furthermore, we will show that this type of systems can exhibit the Painlevé paradox for arbitrary (positive) values of the friction coefficient.

The hopping phenomenon is explained and analyzed using Amontons-Coulomb's law and Newton's restitution law. We acknowledge that these contact laws provide only a crude approximation of the reality of dry friction and impact. However, in most engineering situations, the higher-order information needed for a more accurate description is not available and we have to be content with some moderately precise calculation, accounting at least for the main features of phenomena (Moreau, 1988). The hopping phenomenon occurs generally in complex systems with many degrees of freedom and many contact points. Our aim is to describe the basic mechanism of the hopping motion with a system that is as simple as possible. We will show that a two degree of freedom model (which we call the Frictional Impact Oscillator) with a single contact, assuming Amontons- 
Coulomb's law and Newton's restitution law, is sufficient to describe the essential features of the phenomenon. This relatively simple model shows a complex dynamical behaviour, which might give insight in the dynamics of more complex systems.

The rigid multibody theory (Brogliato, 1999; Glocker, 1995, 2001; Moreau, 1988; Pfeiffer and Glocker, 1996), which assumes instantaneous impact between rigid bodies and Amontons-Coulomb friction model in tangential direction, provides a good approach to study the dynamics when one is interested in the global motion of the system. Mechanical systems with friction and impact, modelled with a rigid multibody approach, belong to the class of hybrid systems (i.e. systems with a mixed continuous and discrete nature in time). The transitions in time from one hybrid mode to another (e.g., from contact to detachment or stick to slip) demand the choice of the next hybrid mode. A rigorous way to perform this search is to formulate the problem as a Linear Complementarity problem (LCP). The solution of the LCP can be nonunique, giving an undeterminate next hybrid mode, and the solution of the LCP may even not exist, which leads to inconsistency in the model. The occurrence of inconsistency in a mechanical rigid multibody system due to friction is known as the Painlevé paradox.

It has been known since the end of the 19th century that the combination of Amontons-Coulomb friction together with the assumption of rigidity can cause inconsistencies for high values of the friction coefficient (Jellet, 1872; Painlevé, 1895a, 1895b, 1905). Painlevé (1895a, 1895b, 1905) considered the sliding motion of rigid objects ${ }^{1}$ in contact with the ground, which have inconsistencies in the sliding mode. The problem of a sliding rod studied by Painlevé became the classical example of a hybrid system with inconsistencies. Lecornu (1905a) proposed velocity jumps to escape from inconsistent modes, often now addressed as impacts without collisions. A number of studies show that inconsistencies occur in the classical Painlevé example when the friction coefficient $\mu$ is larger than 4/3 (Glocker, 1995; Lötstedt, 1981; Mason and Wang, 1988). The classical Painlevé example was recently studied in more detail in (Génot and Brogliato, 1999). New results on how the solution passes singular points in the sliding mode were presented in (Génot and Brogliato, 1999).

The critical value of 4/3 is very large and not likely to occur in physical situations. In (Génot, 1998) an adapted version of the classical Painlevé example was proposed, where the rod has a rounded tip, which lowers the critical friction coefficient to 0.63 (the example with a rounded tip was inspired by Moreau (1986)). The original example studied by Painlevé (1895b), being the contact of a box with the ground, can have a much lower critical friction coefficient for a small radius of gyration (see Appendix A). The occurrence of the Painlevé paradox of a rigid body with a friction rotor was studied in (Wilms and Cohen, 1981, 1997) and a critical friction coefficient was found which can take arbitrary (positive) values depending on other parameters. A similar conclusion is drawn in the present paper.

A model of a hopping finger was studied by Moreau (1986). Stick-slip motion with and without flight phase were reported, as well period-doubled motion, but the occurrence of the Painlevé paradox was not studied. The Frictional Impact Oscillator, presented in the present paper, is slightly different and lends itself better to an analytical investigation of the Painlevé paradox.

Control of the sliding motion of an end-effector of a robot on the surface of an obstacle can be hampered by the same instability phenomena which govern the Frictional Impact Oscillator. It is expected that the results on the Frictional Impact Oscillator, which will be studied in the sequel, can help to design better control schemes for constrained robotic motion.

Unilateral contact laws, as are used in the rigid multibody approach, lead to nonsmooth mathematical models due to their set-valued nature. Bifurcations in smooth systems are well understood (Guckenheimer and Holmes, 1983) but little is known about bifurcations in nonsmooth systems (Leine, 2000). Literature on bifurcations in nonsmooth mechanical systems seems to be divided in two groups:

(1) Bifurcations in systems with friction, which belong to the class of Filippov-systems. Literature on this topic is vast (for instance (Dankowicz and Nordmark, 2000; Galvanetto and Knudsen, 1997; Hensen et al., 2002a, 2002b; Leine and Van Campen, 1999, 2002; Popp et al., 1995; Van de Vrande et al., 1999; Wiercigroch, 1996; Yoshitake and Sueoka, 2000). A general theory for bifurcations in Filippov-systems is not available but attempts to explore in that direction are made (di Bernardo et al., 1999; Leine et al., 2000; Leine, 2000).

(2) Bifurcations in systems with impact (Foale and Bishop, 1994; Ivanov, 1996; Meijaard, 1996; Nordmark, 1997; Peterka, 1996). The impacts are almost always considered to be frictionless and the systems very often contain only a single contact.

Literature on bifurcations in systems with multiple contacts with combined friction and impact is hardly available. An impact oscillator with friction is studied in (Blazejczyk-Okolewska and Kapitaniak, 1996) but the impact and friction are in different contact points for this system and the contact problem is therefore decoupled. Mechanical systems with combined impact and friction are studied in (Leine et al., 2002) and bifurcation diagrams are constructed with the aid of a one-dimensional Poincare

1 The original example studied by Painlevé in (Painlevé, 1895b) is that of a box on an inclined plane. A brief description of the original example of Painlevé is given in Appendix A. The classical example of Painlevé, being a sliding rod, was studied in (Painlevé, 1905). 
map. The topic of bifurcations which are created by the Painlevé paradox has up to now not been addressed in literature and will be studied in the present paper.

The main results on the classical Painlevé example will be briefly discussed in Section 3. The Frictional Impact Oscillator will be studied in Section 4. This model can be simplified which leads to the Simplified Frictional Impact Oscillator (Section 5). Critical friction coefficients of the Painlevé paradox will be derived for both models and numerical results for the periodic solutions will be given. Bifurcations diagrams of the models are presented in Section 6. The notation as well as the methods of (Glocker, 1995; Pfeiffer and Glocker, 1996) are used for the dynamics of rigid multibody systems with impact and friction and are reviewed in Section 2.

\section{Mathematical modeling of impact with friction}

In this section we will briefly review some aspects of a mathematical theory for the dynamics of rigid bodies with completely inelastic frictional impact as has been formulated in (Glocker, 1995, 2001; Pfeiffer and Glocker, 1996). A shorter version of this formulation can be found in (Leine et al., 2002).

For the description on the dynamics of multibody systems it is convenient to introduce four contact sets, which describe the kinematic state of the constraints of the system:

$$
\begin{aligned}
& I_{G}=\left\{1,2, \ldots, n_{G}\right\}, \\
& I_{S}=\left\{i \in I_{G} \mid g_{N i}=0\right\} \quad \text { with } n_{S} \text { elements, } \\
& I_{N}=\left\{i \in I_{S} \mid \dot{g}_{N i}=0\right\} \quad \text { with } n_{N} \text { elements, } \\
& I_{H}=\left\{i \in I_{N} \mid \dot{g}_{T i}=0\right\} \quad \text { with } n_{H} \text { elements, }
\end{aligned}
$$

where $g_{N i}$ and $\dot{g}_{T i}$ denote the normal contact distance and tangential relative velocity of constraint $i$. The set $I_{G}$ consists of $n_{G}$ indices of all constraints, which we want to take into account. $I_{S}$ contains the $n_{S}$ indices of the constraints with vanishing normal distance but arbitrary relative velocity. In the set $I_{N}$ are the $n_{N}$ indices of the potentially active normal constraints, which fulfill the necessary conditions for continuous contact (vanishing normal distance and no relative velocity in normal direction). The set $I_{N}$ contains therefore all indices of slipping or sticking contacts. The $n_{H}$ elements of the set $I_{H}$ correspond to the potentially active constraints in tangential direction (sticking). The sets $I_{S}, I_{N}, I_{H}$ are not constant, because the contact configuration of the dynamical system changes with time due to stick-slip transitions, impact and contact loss.

The dynamics of a multibody system can be expressed for almost all $t$ by the equation of motion

$$
\boldsymbol{M}(t, \boldsymbol{q}) \ddot{\boldsymbol{q}}-\boldsymbol{h}(t, \boldsymbol{q}, \dot{\boldsymbol{q}})-\sum_{i \in I_{S}}\left(\boldsymbol{w}_{N}(t, \boldsymbol{q}) \lambda_{N}+\boldsymbol{w}_{T}(t, \boldsymbol{q}) \lambda_{T}\right)_{i}=\mathbf{0},
$$

where $\boldsymbol{M}$ is the symmetric mass matrix, $\boldsymbol{q}$ the vector of generalized coordinates, $\boldsymbol{h}$ the vector with all smooth elastic, gyroscopic and dissipating generalized forces and $\lambda_{N}$ and $\lambda_{T}$ the vectors with normal and tangential contact forces. The vectors $\boldsymbol{w}_{N}$ and $\boldsymbol{w}_{T}$ are the normal and tangential force directions.

We assume the generalized coordinates $\boldsymbol{q}(t)$ to be absolute continuous functions in time and the generalized velocities $\dot{\boldsymbol{q}}(t)$ to be functions of locally bounded variations, when a solution exists of course. We therefore can define the left and right limits $\dot{\boldsymbol{q}}\left(t^{-}\right)$and $\dot{\boldsymbol{q}}\left(t^{+}\right)$at each time instant $t>t_{0}$ (Brogliato, 1999; Glocker, 2001). Furthermore, we assume the generalized velocities to be right-continuous, i.e. $\dot{\boldsymbol{q}}(t)=\dot{\boldsymbol{q}}\left(t^{+}\right)$. We specify the initial condition at $t=t_{0}$ by $\left(\boldsymbol{q}_{0}, \dot{\boldsymbol{q}}_{0}\right)=\left(\boldsymbol{q}\left(t_{0}\right), \dot{\boldsymbol{q}}\left(t_{0}\right)\right)$. If we allow a first velocity jump to occur at $t_{0}$ then we set $\dot{\boldsymbol{q}}_{0}=\dot{\boldsymbol{q}}\left(t_{0}^{-}\right)$(Moreau, 1988). Typically, the contact forces $\lambda_{N}$ and $\lambda_{T}$ become impulsive when an impact occurs and we have $\dot{\boldsymbol{q}}\left(t^{-}\right) \neq \dot{\boldsymbol{q}}\left(t^{+}\right)$.

More conveniently we put (2.2) in the form

$$
\boldsymbol{M} \ddot{\boldsymbol{q}}-\boldsymbol{h}-\boldsymbol{W}_{N} \lambda_{N}-\boldsymbol{W}_{T} \boldsymbol{\lambda}_{T}=\mathbf{0},
$$

where the dependencies on $t, \boldsymbol{q}, \dot{\boldsymbol{q}}$ have been omitted for brevity and where $\boldsymbol{W}_{N}$ and $\boldsymbol{W}_{T}$ are matrices containing the generalized force directions in normal and tangential direction. The contact distances $g_{N i}$ and $g_{T i}$ are gathered in the vectors $\boldsymbol{g}_{N}$ and $\boldsymbol{g}_{T}$. We can express the contact velocities and accelerations in the generalized accelerations by

$$
\left[\begin{array}{c}
\dot{\boldsymbol{g}}_{N} \\
\dot{\boldsymbol{g}}_{T}
\end{array}\right]=\left[\begin{array}{c}
\boldsymbol{W}_{N}^{\mathrm{T}} \\
\boldsymbol{W}_{T}^{\mathrm{T}}
\end{array}\right] \dot{\boldsymbol{q}}+\left[\begin{array}{c}
\widetilde{\boldsymbol{w}}_{N} \\
\widetilde{\boldsymbol{w}}_{T}
\end{array}\right], \quad\left[\begin{array}{c}
\ddot{\boldsymbol{g}}_{N} \\
\ddot{\boldsymbol{g}}_{T}
\end{array}\right]=\left[\begin{array}{c}
\boldsymbol{W}_{N}^{\mathrm{T}} \\
\boldsymbol{W}_{T}^{\mathrm{T}}
\end{array}\right] \ddot{\boldsymbol{q}}+\left[\begin{array}{c}
\overline{\boldsymbol{w}}_{N} \\
\overline{\boldsymbol{w}}_{T}
\end{array}\right] .
$$

Each closed contact $i \in I_{N}$ is characterized by a vanishing contact distance $g_{N i}$ and normal relative velocity $\dot{g}_{N i}$. Under the assumption of impenetrability $g_{N i} \geqslant 0$, only two situations may occur: 


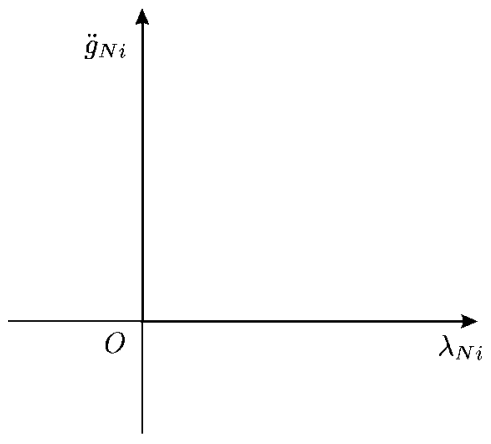

(a) normal direction

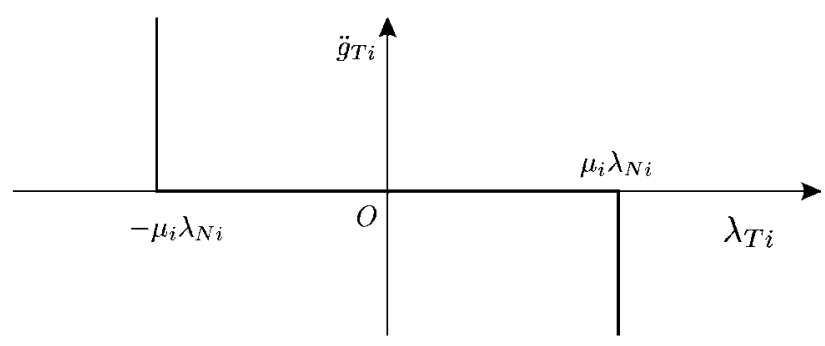

(b) tangential direction

Fig. 2.1. Complementarity of contacts.

$$
\begin{array}{ll}
\ddot{g}_{N i}=0 \wedge \lambda_{N i} \geqslant 0 & \text { (contact is maintained), } \\
\ddot{g}_{N i}>0 \wedge \lambda_{N i}=0 & \text { (detachment). }
\end{array}
$$

From (2.5) we see that the normal contact law shows a complementary behaviour: the product of the contact force and acceleration is always zero:

$$
\ddot{g}_{N i} \lambda_{N i}=0, \quad i \in I_{N} .
$$

The complementary behaviour of the normal contact law is depicted in Fig. 2.1(a) and shows a corner of admissible combinations of $\ddot{g}_{N i}$ and $\lambda_{N i}$.

We assume Amontons-Coulomb law to hold in the tangential direction. For a closed contact $i \in I_{N}$, with friction coefficient $\mu_{i}$, the following three cases are possible:

$$
\begin{array}{ll}
\dot{g}_{T i}=0 \Rightarrow\left|\lambda_{T i}\right| \leqslant \mu_{i} \lambda_{N i} & \text { (sticking), } \\
\dot{g}_{T i}<0 \Rightarrow \lambda_{T i}=+\mu_{i} \lambda_{N i} & \text { (negative sliding), } \\
\dot{g}_{T i}>0 \Rightarrow \lambda_{T i}=-\mu_{i} \lambda_{N i} & \text { (positive sliding), } i \in I_{N} .
\end{array}
$$

To determine the tangential contact force during sticking, one can formulate unilateral laws for sticking contacts. For a closed sticking contact $i \in I_{H}$ the following three cases are possible:

$$
\begin{array}{ll}
\ddot{g}_{T i}=0 \Rightarrow\left|\lambda_{T i}\right| \leqslant \mu_{i} \lambda_{N i} & \text { (remains sticking), } \\
\ddot{g}_{T i}<0 \Rightarrow \lambda_{T i}=+\mu_{i} \lambda_{N i} & \text { (commences negative sliding), } \\
\ddot{g}_{T i}>0 \Rightarrow \lambda_{T i}=-\mu_{i} \lambda_{N i} & \text { (commences positive sliding), } \quad i \in I_{H} .
\end{array}
$$

The normal and tangential contact law lead, together with the equations of motion, to the coupled normal-tangential contact problem for the stick-slip and detachment transitions of the multibody system

$$
\left[\begin{array}{c}
\ddot{\boldsymbol{g}}_{N} \\
\ddot{\boldsymbol{g}}_{H}
\end{array}\right]=\left[\begin{array}{cc}
\boldsymbol{W}_{N}^{\mathrm{T}} \boldsymbol{M}^{-1}\left(\boldsymbol{W}_{N}+\boldsymbol{W}_{G} \boldsymbol{\mu}_{G}\right) & \boldsymbol{W}_{N}^{\mathrm{T}} \boldsymbol{M}^{-1} \boldsymbol{W}_{H} \\
\boldsymbol{W}_{H}^{\mathrm{T}} \boldsymbol{M}^{-1}\left(\boldsymbol{W}_{N}+\boldsymbol{W}_{G} \boldsymbol{\mu}_{G}\right) & \boldsymbol{W}_{H}^{\mathrm{T}} \boldsymbol{M}^{-1} \boldsymbol{W}_{H}
\end{array}\right]\left[\begin{array}{c}
\lambda_{N} \\
\lambda_{H}
\end{array}\right]+\left[\begin{array}{c}
\boldsymbol{W}_{N}^{\mathrm{T}} \boldsymbol{M}^{-1} \boldsymbol{h}+\overline{\boldsymbol{w}}_{N} \\
\boldsymbol{W}_{H}^{\mathrm{T}} \boldsymbol{M}^{-1} \boldsymbol{h}+\overline{\boldsymbol{w}}_{H}
\end{array}\right]
$$

with $^{2}$

$$
\begin{array}{ll}
0 \leqslant \ddot{\boldsymbol{g}}_{N} \perp \lambda_{N} \geqslant 0 & \in I_{N}, \\
\lambda_{H} \in-\boldsymbol{\mu}_{H} \lambda_{N} \operatorname{diag}\left(\partial\left|\ddot{\boldsymbol{g}}_{H}\right|\right) & \in I_{H},
\end{array}
$$

where

${ }^{2}$ The notation $\boldsymbol{a} \perp \boldsymbol{b}$ means that $\boldsymbol{a}$ stands perpendicular to $\boldsymbol{b}$, i.e. $\boldsymbol{a}^{\mathrm{T}} \boldsymbol{b}=0$. It follows therefore from the complementarity conditions $\mathbf{0} \leqslant \boldsymbol{a} \perp \boldsymbol{b} \geqslant \mathbf{0}$, that if $a_{i}>0$ then $b_{i}=0$ and vice versa. 


$$
\begin{aligned}
& \boldsymbol{W}_{G}=\boldsymbol{w}_{T i}, \quad i \in I_{N} \backslash I_{H}, \\
& \boldsymbol{g}_{H}=g_{T i}, \quad \lambda_{H}=\lambda_{T i}, \quad \boldsymbol{W}_{H}=\boldsymbol{w}_{T i}, \quad \overline{\boldsymbol{w}}_{H}=\bar{w}_{T i}, \quad i \in I_{H}, \\
& \boldsymbol{\mu}_{H} \in \mathbb{R}^{n_{H} \times n_{N}}, \quad \boldsymbol{\mu}_{G} \in \mathbb{R}^{n_{N}-n_{H} \times n_{N}},
\end{aligned}
$$

and where the subdifferential of convex analysis $\partial|x|=\operatorname{Sign}(x)$ has been used. Equations (2.9) can be transformed into an LCP as has been formulated in (Glocker, 1995; Pfeiffer and Glocker, 1996).

If an impact occurs, then we generally have a discontinuity in the generalized velocities $\dot{\boldsymbol{q}}$. The impact is assumed to begin at a time $t^{-}$and to end at a time $t^{+}$. The time difference $t^{+}-t^{-}$is assumed to be "infinitely small" 3 in the rigid multibody approach. The equation of motion is integrated over the impact time:

$$
\boldsymbol{M}\left(\dot{\boldsymbol{q}}\left(t^{+}\right)-\dot{\boldsymbol{q}}\left(t^{-}\right)\right)=\left[\begin{array}{ll}
\boldsymbol{W}_{N} & \boldsymbol{W}_{T}
\end{array}\right]\left[\begin{array}{l}
\boldsymbol{\Lambda}_{N} \\
\boldsymbol{\Lambda}_{T}
\end{array}\right],
$$

which yields the velocity jump $\dot{\boldsymbol{q}}\left(t^{+}\right)-\dot{\boldsymbol{q}}\left(t^{-}\right)$as a function of the impulses $\boldsymbol{\Lambda}_{N}$ and $\boldsymbol{\Lambda}_{T}$ in normal and tangential direction defined by

$$
\Lambda_{N i}=\lim _{t^{+} \rightarrow t^{-}} \int_{t^{-}}^{t^{+}} \lambda_{N i} \mathrm{~d} t, \quad \Lambda_{T i}=\lim _{t^{+} \rightarrow t^{-}} \int_{t^{-}}^{t^{+}} \lambda_{T i} \mathrm{~d} t, \quad i \in I_{S} .
$$

Due to the unilateral character of the contact constraint only nonnegative normal contact forces are possible, $\lambda_{N i} \geqslant 0$, which results in nonnegative values of the normal impulses $\Lambda_{N i} \geqslant 0$. At the end of the completely inelastic impact the approaching process of the bodies has to be completed. Thus negative values of the contact velocities are forbidden, $\dot{g}_{N i}\left(t^{+}\right) \geqslant 0$. If an impulse is transferred $\left(\Lambda_{N i}>0\right)$, then the corresponding contact participates in the impact and $\dot{g}_{N i}\left(t^{+}\right)=0$. If no impulse is transferred $\left(\Lambda_{N C i}=0\right)$, then the corresponding constraint is superfluous and we allow velocities $\dot{g}_{N i}\left(t^{+}\right) \geqslant 0$. The impact law in normal direction is therefore expressed by the complementarity condition

$$
\Lambda_{N i} \geqslant 0, \quad \dot{g}_{N i}\left(t^{+}\right) \geqslant 0, \quad \Lambda_{N i} \dot{g}_{N i}\left(t^{+}\right)=0, \quad i \in I_{S} .
$$

Possible stick-slip transitions during the collision with reversed sliding prevent an analytical integration of Coulomb's friction law (2.8) over the impact time interval. However, we state the tangential impact law as

$$
\begin{aligned}
& \dot{g}_{T i}\left(t^{+}\right)=0 \Rightarrow\left|\Lambda_{T i}\right| \leqslant \mu_{i} \Lambda_{N i} \quad \text { (sticking), } \\
& \dot{g}_{T i}\left(t^{+}\right)<0 \Rightarrow \Lambda_{T i}=+\mu_{i} \Lambda_{N i} \quad \text { (negative sliding), } \\
& \dot{g}_{T i}\left(t^{+}\right)>0 \Rightarrow \Lambda_{T i}=-\mu_{i} \Lambda_{N i} \quad \text { (positive sliding), } \quad i \in I_{S},
\end{aligned}
$$

with the remark that (2.15) coincides with Coulomb's friction law (2.8) in the cases of continuous sliding during the impact and of arbitrary transitions to sticking at the end of the impact. Only events of reversed sliding or transitions from sticking to sliding with a sliding phase at the end of the impact are different from Coulomb's law (Glocker, 1995; Moreau, 1988; Pfeiffer and Glocker, 1996).

Evaluating the contact velocities (2.4) at $t^{+}$and $t^{-}$gives

$$
\left[\begin{array}{l}
\dot{\boldsymbol{g}}_{N}\left(t^{+}\right) \\
\dot{\boldsymbol{g}}_{T}\left(t^{+}\right)
\end{array}\right]=\left[\begin{array}{c}
\boldsymbol{W}_{N}^{\mathrm{T}} \\
\boldsymbol{W}_{T}^{\mathrm{T}}
\end{array}\right]\left(\dot{\boldsymbol{q}}\left(t^{+}\right)-\dot{\boldsymbol{q}}\left(t^{-}\right)\right)+\left[\begin{array}{l}
\dot{\boldsymbol{g}}_{N}\left(t^{-}\right) \\
\dot{\boldsymbol{g}}_{T}\left(t^{-}\right)
\end{array}\right] \in \mathbb{R}^{2 n_{S}} .
$$

Substitution of (2.12) in (2.16) together with the contact laws (2.14) and (2.15) gives the set of equations

$$
\left[\begin{array}{c}
\dot{\boldsymbol{g}}_{N}\left(t^{+}\right) \\
\dot{\boldsymbol{g}}_{T}\left(t^{+}\right)
\end{array}\right]=\left[\begin{array}{ll}
\boldsymbol{W}_{N}^{\mathrm{T}} \boldsymbol{M}^{-1} \boldsymbol{W}_{N} & \boldsymbol{W}_{N}^{\mathrm{T}} \boldsymbol{M}^{-1} \boldsymbol{W}_{T} \\
\boldsymbol{W}_{T}^{\mathrm{T}} \boldsymbol{M}^{-1} \boldsymbol{W}_{N} & \boldsymbol{W}_{T}^{\mathrm{T}} \boldsymbol{M}^{-1} \boldsymbol{W}_{T}
\end{array}\right]\left[\begin{array}{c}
\boldsymbol{\Lambda}_{N} \\
\boldsymbol{\Lambda}_{T}
\end{array}\right]+\left[\begin{array}{l}
\dot{\boldsymbol{g}}_{N}\left(t^{-}\right) \\
\dot{\boldsymbol{g}}_{T}\left(t^{-}\right)
\end{array}\right]
$$

with

$$
\begin{array}{ll}
0 \leqslant \dot{\boldsymbol{g}}_{N}\left(t^{+}\right) \perp \boldsymbol{\Lambda}_{N} \geqslant 0 & \in I_{S}, \\
\boldsymbol{\Lambda}_{T} \in-\boldsymbol{\mu}_{S} \boldsymbol{\Lambda}_{N} \operatorname{diag}\left(\partial\left|\dot{\boldsymbol{g}}_{T}\left(t^{+}\right)\right|\right) & \in I_{S},
\end{array}
$$

where $\mu_{S}=\operatorname{diag}\left(\mu_{i}\right), i \in I_{S}$. The formulation of the coupled normal-tangential contact problem for completely inelastic impact is given by (2.17) and is usually solved by reformulating it as an LCP.

\footnotetext{
3 Mathematically more correct is to consider the impact as a singleton, i.e. a point in time, and the equation of motion as a measure differential equation, which is beyond the scope of this paper (see (Glocker, 2000; Moreau, 1988)).
} 
In the sequel we will use an event-driven integration scheme to obtain solutions $\boldsymbol{q}(t)$ of unilaterally constrained mechanical systems. The equation of motion (2.3) for given index sets is numerically integrated until an impact, stick-slip or detachment event occurs. If the event is an impact event, then the impact equations (2.17) have to be solved, after which the new generalized velocities $\dot{\boldsymbol{q}}$ are known. Subsequently, Eq. (2.9) on acceleration level have to be solved, because the impact might cause stickslip transitions or detachment of other contacts. The new accelerations $\ddot{\boldsymbol{q}}$ are known after having solved (2.9). The new index sets can then be setup and a new smooth integration phase can start. Basically any ODE-solver can be used for the integration of the smooth phase as long as the solver supports event detection.

The event-driven integration scheme complies with a certain solution concept. The solution remains in a smooth mode as long as some indicator inequalities are met (e.g., $\left|\lambda_{T}\right| \leqslant \lambda_{N}$ in the stick mode, $g_{N} \geqslant 0$ in the unconstrained mode). If an indicator function is violated, then an event occurs, and Eqs. (2.17) and (2.9) are solved which are basically LCPs. The LCPs derived from (2.17) and (2.9) determine the mode change, i.e. they indicate the next mode. The solution of the LCPs can be nonunique, indicating nonuniqueness of the next mode and therefore nonuniqueness of the solution $\boldsymbol{q}(t)$. The solution of the LCPs can also be nonexistent, implying that there is no continuation possible in any mode, and therefore nonexistence of the solution $\boldsymbol{q}(t)$.

\section{The classical Painlevé example}

In this section we will briefly summarize some results on the classical Painlevé problem of a sliding rigid rod. The rigid body formulation of the model is taken from (Glocker, 1995) and the main results from (Brogliato, 1999; Génot and Brogliato, 1999).

Consider a rigid homogeneous slender rod with mass $m$, length $2 s$ and inertia $J_{S}=\frac{1}{3} m s^{2}$ (see Fig. 3.1). The rod is sliding with one tip over a rigid ground. Amontons-Coulomb friction model is supposed to hold at the contact point with constant friction coefficient $\mu>0$. The system has three degrees of freedom, which we gather in a vector of generalized coordinates $\boldsymbol{q}=\left[\begin{array}{lll}x & y & \varphi\end{array}\right]^{\mathrm{T}}$. The normal contact distance and tangential contact velocity are

$$
g_{N}=y-s \sin \varphi, \quad \dot{g}_{T}=\dot{x}-s \dot{\varphi} \sin \varphi .
$$

The system matrices of the Painlevé problem become

$$
\begin{aligned}
& \boldsymbol{M}=\left[\begin{array}{ccc}
m & 0 & 0 \\
0 & m & 0 \\
0 & 0 & J_{S}
\end{array}\right], \quad \boldsymbol{h}=\left[\begin{array}{c}
0 \\
-m g \\
0
\end{array}\right], \quad \boldsymbol{W}_{N}=\left[\begin{array}{c}
0 \\
1 \\
-s \cos \varphi
\end{array}\right], \quad \boldsymbol{W}_{T}=\left[\begin{array}{c}
1 \\
0 \\
-s \sin \varphi
\end{array}\right], \\
& \widetilde{\boldsymbol{w}}_{N}=\widetilde{\boldsymbol{w}}_{T}=0, \quad \overline{\boldsymbol{w}}_{N}=s \dot{\varphi}^{2} \sin \varphi, \quad \overline{\boldsymbol{w}}_{T}=-s \dot{\varphi}^{2} \cos \varphi .
\end{aligned}
$$

It is relatively easy to show that a dynamic frictional catastrophe can occur in this simple system. We assume that the rod is sliding over the ground in forward direction, i.e., $g_{N}=0, \dot{g}_{N}=0$ and $\dot{g}_{T}>0$. It therefore holds that $\lambda_{T}=-\mu \lambda_{N}$. The equations of motion become in the forward sliding mode:

$$
m \ddot{x}=-\mu \lambda_{N}, \quad m \ddot{y}=-m g+\lambda_{N}, \quad J_{S} \ddot{\varphi}=s(-\cos \varphi+\mu \sin \varphi) \lambda_{N} .
$$

The rod remains in sliding contact with the ground when $\ddot{g}_{N}=0$ and will detach when $\ddot{g}_{N}>0$, with

$$
\ddot{g}_{N}=\ddot{y}-s \cos \varphi \ddot{\varphi}+s \dot{\varphi}^{2} \sin \varphi .
$$

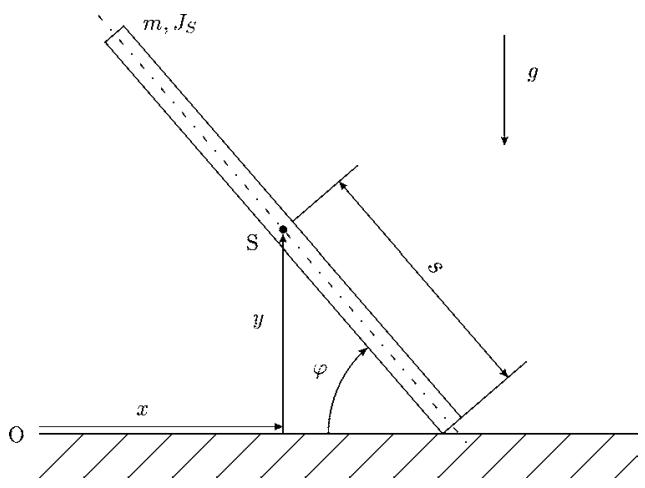

Fig. 3.1. The classical Painlevé example. 
The normal contact acceleration becomes in the forward sliding mode (substituting (3.4) in (3.5)):

$$
\ddot{g}_{N}=A \lambda_{N}+b,
$$

with

$$
A(\varphi)=\frac{1}{m}(1+3 \cos \varphi(\cos \varphi-\mu \sin \varphi)), \quad b(\varphi, \dot{\varphi})=s \dot{\varphi}^{2} \sin \varphi-g .
$$

Note that $A$ is a function of $\varphi$ and $\mu$ while $b$ is function of $\varphi$ and $\dot{\varphi}$. Between the normal contact acceleration $\ddot{g}_{N}$ and the normal contact force $\lambda_{N}$ the following complementarity conditions hold:

$$
0 \leqslant \ddot{g}_{N} \perp \lambda_{N} \geqslant 0 .
$$

The complementarity conditions (3.8) express that if the contact detaches, $\ddot{g}_{N}>0$, then it must hold that $\lambda_{N}=0$, while if the normal contact force is positive $\lambda_{N}>0$ the contact remains closed $\ddot{g}_{N}=0$. The linear equation (3.6) together with the complementarity conditions (3.8) gives a scalar Linear Complementarity Problem (LCP) for the detachment of the rod in the forward sliding mode. The scalar LCP with $\ddot{g}_{N}=A \lambda_{N}+b, 0 \leqslant \ddot{g}_{N} \perp \lambda_{N} \geqslant 0$ has a unique solution for $A>0$, two solutions for $A<0 \wedge b>0$, no solution for $A<0 \wedge b<0$ or infinitely many solutions for $A=b=0$ (hyperstatic case). The solutions of the LCP are indicated in Table 3.1. The occurrence of inconsistency (the LCP has no solution) and indeterminacy (nonuniqueness of solutions) is called the Painlevé paradox (Génot and Brogliato, 1999).

The Painlevé paradox will occur when $A(\varphi)$ becomes negative, which happens for large enough values of $\mu$. The critical value of $\mu$ for which $A(\varphi)=0$ occurs at $\mu=\mu_{c}(\varphi)$ with

$$
\mu_{c}(\varphi)=\frac{1+3 \cos ^{2} \varphi}{3 \sin \varphi \cos \varphi} .
$$

Table 3.1

LCP solutions

\begin{tabular}{llll}
\hline$A$ & $b$ & Number of solutions & \multicolumn{1}{c}{ LCP solutions } \\
\hline$A>0$ & $b>0$ & unique solution & $\lambda_{N}=0 \wedge \ddot{g}_{N}=b$ \\
$A>0$ & $b<0$ & unique solution & $\lambda_{N}=-\frac{b}{A} \wedge \ddot{g}_{N}=0$ \\
$A<0$ & $b>0$ & two solutions & $\lambda_{N}=-\frac{b}{A} \wedge \ddot{g}_{N}=0$ or $\lambda_{N}=0 \wedge \ddot{g}_{N}=b$ \\
$A<0$ & $b<0$ & no solution & $\emptyset$ \\
\hline
\end{tabular}

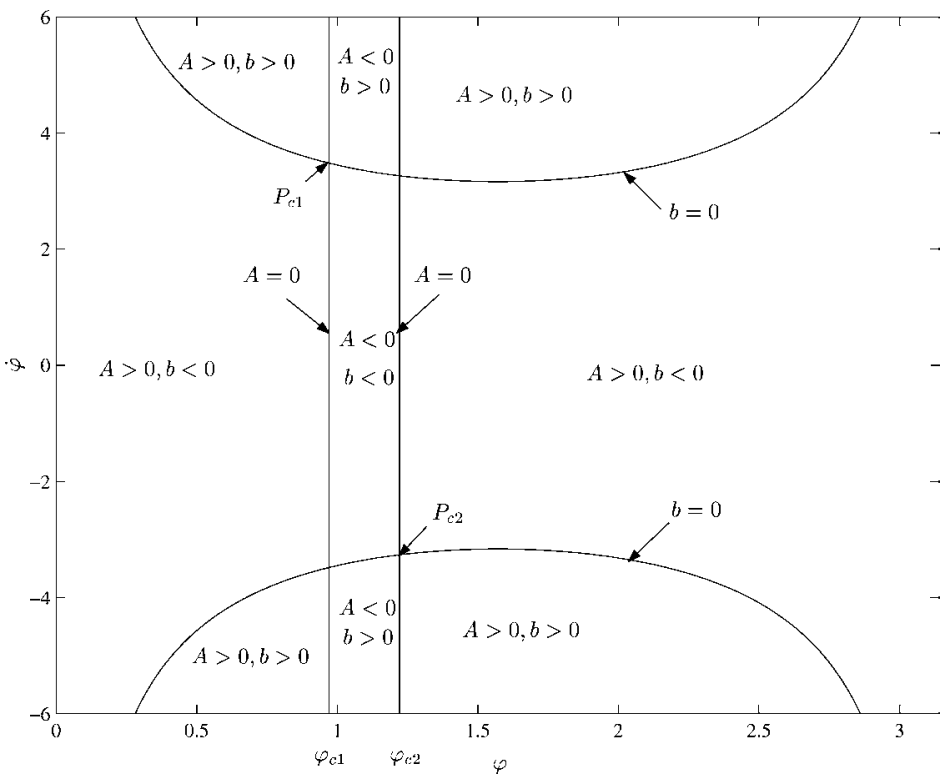

Fig. 3.2. The $(\varphi, \dot{\varphi})$-plane of the classical Painlevé problem (Génot and Brogliato, 1999). 
The critical friction $\mu_{c}(\varphi)$ coefficient is minimal for $\varphi=\arctan 2$, giving $\mu_{c \text { min }}=4 / 3$. This minimum critical friction coefficient changes for other ratios of $m s^{2} / J_{S}$ but will always be larger than 1 for $m>0$ and $J_{S}>0$. For $\mu \geqslant \mu_{c \text { min }}$ there exists an interval of $\varphi_{c 1}<\varphi<\varphi_{c 2}$ for which $A<0$ with

$$
\varphi_{c 1}=\arctan \left(\frac{3 \mu-\sqrt{9 \mu^{2}-16}}{2}\right), \quad \varphi_{c 2}=\arctan \left(\frac{3 \mu+\sqrt{9 \mu^{2}-16}}{2}\right) .
$$

The $(\varphi, \dot{\varphi})$-plane is depicted in Fig. 3.2 together with the four different LCP modes for detachment in forward sliding. A detailed study of the singular points $P_{c 1}$ and $P_{c 2}$ (Fig. 3.2) is given in (Génot and Brogliato, 1999). It is proven in (Génot and Brogliato, 1999) that the solution can reach the singular points $P_{c 1}$ and $P_{c 2}$, depending on the friction coefficient, either with a finite contact force or with a contact force reaching infinite values.

In the sequel we will present a model, consisting of a body with normal and tangential degrees of freedom, which exhibits the Painlevé paradox. We will prove that a minimum critical friction coefficient for the Painlevé paradox is possible which can be arbitrary small depending an some mass ratio. Furthermore, we will analyze the bifurcations of this model and will show that a figure like Fig. 3.2 can be a very useful tool to analyze bifurcations caused by Painlevé paradoxes.

\section{The Frictional Impact Oscillator}

A human finger which is pushed over a table may exhibit periodic motion with stick and slip phases. When the friction between the finger-tip and the table is sufficiently high, even periodic motion may be observed with phases where the finger is not in contact with the table, i.e. the finger detaches from the table causing a 'flight' phase after which an impact occurs to another phase of the periodic motion. In this section we will study a simple system, called the Frictional Impact Oscillator, which exhibits the same phenomenon. The system is low-dimensional ( 2 degrees-of-freedom) but shows rich dynamic behaviour, being complicated by the Painlevé paradox.

A similar system was studied by Moreau (1986). The system of Moreau consists of a bar with a rounded tip which is mounted elastically in horizontal and vertical direction, which leads to a 3 degree-of-freedom system. The horizontal compliance is not essential for the phenomenon and is neglected in the present study, which facilitates the analysis.

\subsection{Model}

The Frictional Impact Oscillator is depicted in Fig. 4.1. The system consists of a mass-spring-damper system (with coordinate $y$ and constants $m_{2}, k, c$ ), which can be looked upon as the 'hand', robot arm or supporting structure. A massless rigid bar, representing the 'finger' or end-effector of a robot, is attached by a hinge to the 'hand' or robot arm, respectively. The hinge can only move in the vertical direction by a displacement $y$. The bar is mounted at the hinge by a rotational spring and dashpot $\left(k_{\varphi}, c_{\varphi}\right)$. A mass $m_{1}$ is located at the tip of the bar. The springs $k$ and $k_{\varphi}$ are unstressed when $y=0$ and $\varphi=\varphi_{0}$, respectively. The tip of the bar can make contact with a belt, which is moving at constant velocity $v_{\mathrm{dr}}$. The contact is regarded

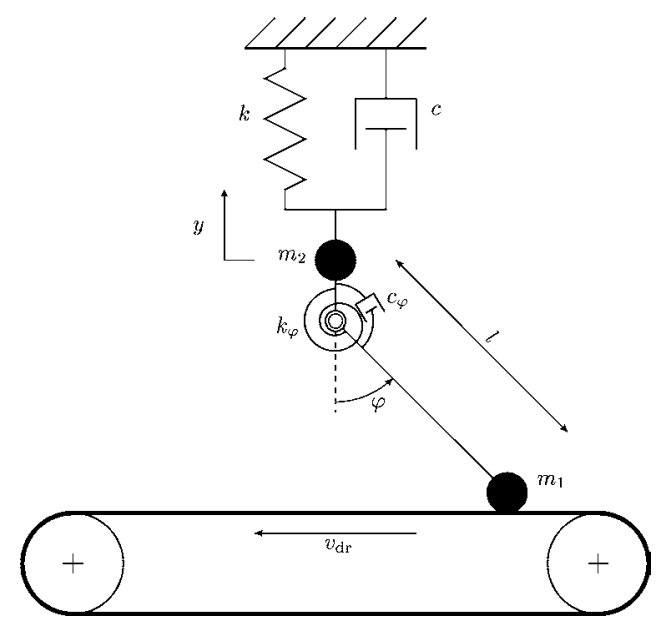

Fig. 4.1. The Frictional Impact Oscillator. 
to be completely inelastic but frictional $(\mu>0)$. Pure Amontons-Coulomb friction is assumed without Stribeck effect ( $\mu$ is not dependent on the relative velocity). The normal contact distance and tangential contact velocity are

$$
g_{N}=l(1-\cos \varphi)+y, \quad \dot{g}_{T}=l \dot{\varphi} \cos \varphi+v_{\mathrm{dr}} .
$$

The rotation of the bar $\varphi$ and the vertical displacement of the hinge $y$ can be gathered in a vector of generalized coordinates $\boldsymbol{q}=\left[\begin{array}{ll}\varphi & y\end{array}\right]^{\mathrm{T}}$. The kinetic and potential energy of the system can be expressed by

$$
\begin{aligned}
T & =\frac{1}{2} m_{1} l^{2} \dot{\varphi}^{2}+m_{1} l \sin \varphi \dot{y} \dot{\varphi}+\frac{1}{2}\left(m_{1}+m_{2}\right) \dot{y}^{2}, \\
V & =\frac{1}{2} k_{\varphi}\left(\varphi-\varphi_{0}\right)^{2}+\frac{1}{2} k y^{2}+m_{1} g(l-l \cos \varphi+y)+m_{2} g y .
\end{aligned}
$$

Lagrange's equations of motion can be put in the form of (2.3). The system matrices and vectors of the Frictional Impact Oscillator become

$$
\begin{aligned}
& \boldsymbol{M}=\left[\begin{array}{cc}
m_{1} l^{2} & m_{1} l \sin \varphi \\
m_{1} l \sin \varphi & m_{1}+m_{2}
\end{array}\right], \quad \boldsymbol{h}=\left[\begin{array}{c}
-k_{\varphi}\left(\varphi-\varphi_{0}\right)-c_{\varphi} \dot{\varphi}-m_{1} g l \sin \varphi \\
-k y-c \dot{y}-\left(m_{1}+m_{2}\right) g-m_{1} l \cos \varphi \dot{\varphi}^{2}
\end{array}\right], \\
& \boldsymbol{W}_{N}=\left[\begin{array}{c}
l \sin \varphi \\
1
\end{array}\right], \quad \widetilde{\boldsymbol{w}}_{N}=0, \quad \overline{\boldsymbol{w}}_{N}=l \dot{\varphi}^{2} \cos \varphi, \\
& \boldsymbol{W}_{T}=\left[\begin{array}{c}
l \cos \varphi \\
0
\end{array}\right], \quad \tilde{\boldsymbol{w}}_{T}=v_{\mathrm{dr}}, \quad \overline{\boldsymbol{w}}_{T}=-l \dot{\varphi}^{2} \sin \varphi .
\end{aligned}
$$

\subsection{Forward slip, $\dot{g}_{T}>0$}

The motion of the system in the forward slip mode is constraint with $\lambda_{N} \geqslant 0$ and $\lambda_{T}=-\mu \lambda_{N}$. The equations of motion become

$$
\begin{aligned}
& m_{1} l^{2} \ddot{\varphi}+m_{1} l \sin \varphi \ddot{y}+c_{\varphi} \dot{\varphi}+k_{\varphi}\left(\varphi-\varphi_{0}\right)=l(\sin \varphi-\mu \cos \varphi) \lambda_{N}-m_{1} g l \sin \varphi, \\
& m_{1} l \sin \varphi \ddot{\varphi}+\left(m_{1}+m_{2}\right) \ddot{y}+c \dot{y}+k y+m_{1} l \cos \varphi \dot{\varphi}^{2}=\lambda_{N}-\left(m_{1}+m_{2}\right) g .
\end{aligned}
$$

Elimination of $\lambda_{N}$ together with the conditions $g_{N}=\dot{g}_{N}=\ddot{g}_{N}=0$ gives a second-order differential equation

$$
\mathcal{M}(\varphi) \ddot{\varphi}+\mathcal{D}(\varphi) \dot{\varphi}^{2}+\mathcal{C}(\varphi) \dot{\varphi}+\mathcal{F}(\varphi)=0,
$$

with

$$
\begin{aligned}
& \mathcal{M}(\varphi)=\left(m_{1} \cos ^{2} \varphi+m_{2} \sin \varphi(\sin \varphi-\mu \cos \varphi)\right) l^{2}, \\
& \mathcal{D}(\varphi)=\left(\left(-m_{1}+m_{2}\right) \sin \varphi \cos \varphi-m_{2} \mu \cos ^{2} \varphi\right) l^{2}, \\
& \mathcal{C}(\varphi)=c_{\varphi}+c l^{2} \sin \varphi(\sin \varphi-\mu \cos \varphi), \\
& \mathcal{F}(\varphi)=k_{\varphi}\left(\varphi-\varphi_{0}\right)+k l^{2}(1-\cos \varphi)(\sin \varphi-\mu \cos \varphi)+\left(\left(m_{1}+m_{2}\right) \mu \cos \varphi-m_{2} \sin \varphi\right) l g .
\end{aligned}
$$

The system can have (multiple) equilibria $\varphi_{\mathrm{eq}}$ in the forward sliding mode, which obey

$$
\mathcal{F}\left(\varphi_{\text {eq }}\right)=0
$$

and a nonzero contact force

$$
\lambda_{N}=-k l\left(1-\cos \varphi_{\mathrm{eq}}\right)+\left(m_{1}+m_{2}\right) g \geqslant 0 .
$$

If the condition

$$
\mathcal{K}(\varphi)=\frac{\partial \mathcal{F}(\varphi)}{\partial \varphi}>0 \quad \forall \varphi
$$

holds with

$$
\frac{\partial \mathcal{F}}{\partial \varphi}=k_{\varphi}+k l^{2}((1-\cos \varphi)(\cos \varphi+\mu \sin \varphi)+\sin \varphi(\sin \varphi-\mu \cos \varphi))-l g\left(\left(m_{1}+m_{2}\right) \mu \sin \varphi+m_{2} \cos \varphi\right)
$$

then at most one equilibrium in the forward sliding mode exists. This is a sufficient condition for $\mathcal{F}(\varphi)$ to have at most one zero but not a necessary condition. We assume in the forthcoming that $k_{\varphi}$ is chosen large enough that indeed $\mathcal{K}(\varphi)>0$ for all 
$\varphi$ of interest. This means that the unique equilibrium $\varphi_{\mathrm{eq}}$, if it exists and obeys $\lambda_{N}>0$, must be close to $\varphi_{0}$. The equilibrium is approximately located at

$$
\varphi_{\mathrm{eq}} \approx \varphi_{0}-\frac{\mathcal{F}\left(\varphi_{0}\right)}{\mathcal{K}\left(\varphi_{0}\right)}
$$

The equation of motion for $\mathcal{M}(\varphi) \neq 0$ can be written in first-order form as

$$
\dot{\varphi}=\psi, \quad \dot{\psi}=-\mathcal{M}(\varphi)^{-1}\left(\mathcal{D}(\varphi) \psi^{2}+\mathcal{C}(\varphi) \psi+\mathcal{F}(\varphi)\right) .
$$

Linearization around the equilibrium $(\xi, \psi)=(0,0)$ with $\xi=\varphi-\varphi_{\text {eq }}$ gives

$$
\dot{\xi}=\psi, \quad \dot{\psi}=-\left(\mathcal{M}\left(\varphi_{\mathrm{eq}}\right)^{-1}-\mathcal{M}\left(\varphi_{\mathrm{eq}}\right)^{-2} \mathcal{M}^{\prime}\left(\varphi_{\mathrm{eq}}\right) \xi\right)\left(\mathcal{C}\left(\varphi_{\mathrm{eq}}\right) \psi+\mathcal{F}\left(\varphi_{\mathrm{eq}}\right)+\mathcal{K}\left(\varphi_{\mathrm{eq}}\right) \xi\right)+\text { H.O.T. }
$$

with $\mathcal{M}^{\prime}(\varphi)=\partial \mathcal{M} / \partial \varphi$. It should be noted that $\mathcal{F}\left(\varphi_{\text {eq }}\right)=0$. The eigenvalue problem therefore yields

$$
\mathcal{M}\left(\varphi_{\mathrm{eq}}\right) \lambda^{2}+\mathcal{C}\left(\varphi_{\mathrm{eq}}\right) \lambda+\mathcal{K}\left(\varphi_{\mathrm{eq}}\right)=0 .
$$

The equilibrium is stable if $\mathcal{M}\left(\varphi_{\mathrm{eq}}\right)>0, \mathcal{C}\left(\varphi_{\mathrm{eq}}\right)>0$ and $\mathcal{K}\left(\varphi_{\mathrm{eq}}\right)>0$ or $\mathcal{M}\left(\varphi_{\mathrm{eq}}\right)<0, \mathcal{C}\left(\varphi_{\mathrm{eq}}\right)<0$ and $\mathcal{K}\left(\varphi_{\mathrm{eq}}\right)<0$. Note that the friction coefficient $\mu$ can cause a negative feedback of the damping forces. The equilibrium undergoes a Hopf bifurcation for $\mathcal{M}\left(\varphi_{\mathrm{eq}}\right)>0, \mathcal{K}\left(\varphi_{\mathrm{eq}}\right)>0$ when the friction coefficient $\mu$ passes the critical value

$$
\mu_{d}=\tan \varphi_{\mathrm{eq}}+\frac{c_{\varphi}}{c l^{2} \sin \varphi_{\mathrm{eq}} \cos \varphi_{\mathrm{eq}}},
$$

for which $\mathcal{C}\left(\varphi_{\mathrm{eq}}\right)=0 .{ }^{4}$ The value of $\mathcal{M}\left(\varphi_{\mathrm{eq}}\right)$ becomes zero when

$$
\mu=\tan \varphi_{\mathrm{eq}}+\frac{m_{1}}{m_{2}} \frac{1}{\tan \varphi_{\mathrm{eq}}},
$$

which is in fact the Painlevé paradox.

\subsection{Painlevé paradox}

In this subsection we will study the Painlevé paradox in the forward and backward sliding mode with similar techniques as presented in (Génot and Brogliato, 1999).

The solution remains in the forward sliding mode if $\dot{g}_{T}>0$ and $\ddot{g}_{N}=0$ and in the backward sliding mode if $\dot{g}_{T}<0$ and $\ddot{g}_{N}=0$. The body detaches from the belt when $\ddot{g}_{N}>0$. For the normal contact acceleration $\ddot{g}_{N}$ holds:

$$
\ddot{g}_{N}=l \sin \varphi \ddot{\varphi}+\ddot{y}+l \cos \varphi \dot{\varphi}^{2} .
$$

Substituting (2.3) in (2.4) together with $g_{N}=\dot{g}_{N}=0$ gives

$$
\ddot{g}_{N}= \begin{cases}A^{+} \lambda_{N}+b, & \dot{g}_{T}>0, \\ A^{-} \lambda_{N}+b, & \dot{g}_{T}<0\end{cases}
$$

with

$$
\begin{aligned}
A^{+}(\varphi) & =\boldsymbol{W}_{N}^{\mathrm{T}} \boldsymbol{M}^{-1}\left(\boldsymbol{W}_{N}-\mu \boldsymbol{W}_{T}\right)=\frac{\cos ^{2} \varphi}{N(\varphi)}\left(1+\frac{m_{2}}{m_{1}} \tan \varphi(\tan \varphi-\mu)\right), \\
A^{-}(\varphi) & =\boldsymbol{W}_{N}^{\mathrm{T}} \boldsymbol{M}^{-1}\left(\boldsymbol{W}_{N}+\mu \boldsymbol{W}_{T}\right)=\frac{\cos ^{2} \varphi}{N(\varphi)}\left(1+\frac{m_{2}}{m_{1}} \tan \varphi(\tan \varphi+\mu)\right), \\
b(\varphi, \dot{\varphi}) & =\boldsymbol{W}_{N}^{\mathrm{T}} \boldsymbol{M}^{-1} \boldsymbol{h}+\overline{\boldsymbol{w}}_{N} \\
& =\frac{\cos ^{2} \varphi}{N(\varphi)}\left(-\frac{m_{2}}{m_{1}} \frac{\tan \varphi}{l \cos \varphi}\left(c_{\varphi} \dot{\varphi}+k_{\varphi}\left(\varphi-\varphi_{0}\right)\right)+c l \sin \varphi \dot{\varphi}+k l(1-\cos \varphi)+\frac{m_{2} l}{\cos \varphi} \dot{\varphi}^{2}\right)-g
\end{aligned}
$$

and

$$
N(\varphi)=m_{1} \cos ^{2} \varphi+m_{2} .
$$

\footnotetext{
${ }^{4}$ If $\mu$ passes $\mu_{d}$, then it follows that $\mathcal{C}\left(\varphi_{\mathrm{eq}}\right)$ changes sign. A pair of complex conjugated eigenvalues move therefore through the imaginary axis, implying a Hopf bifurcation.
} 
The notation $A^{+}$is used to denote the value of $A$ in the forward sliding mode, while $A^{-}$will be used for the backward sliding mode. Note that $A^{ \pm}$are functions of $\varphi$ and $\mu$, and $b$ is a function of $\varphi$ and $\dot{\varphi}$. The linear equation (4.18) gives together with the complementarity conditions

$$
0 \leqslant \ddot{g}_{N} \perp \lambda_{N} \geqslant 0
$$

a scalar Linear Complementarity Problem for the detachment in the forward and backward sliding mode.

The Painlevé paradox occurs in the forward sliding mode when $A^{+}(\varphi)$ becomes negative, leading to inconsistency or indeterminacy of the forward sliding mode (depending on the sign of $b(\varphi, \dot{\varphi})$ ). The value $A^{+}(\varphi)$ can become negative for sufficiently large values of the friction coefficient $\mu$. The critical value occurs at $\mu=\mu_{c}^{+}(\varphi)$ for which $A^{+}(\varphi)=0$,

$$
\mu_{c}^{+}(\varphi)=\tan \varphi+\frac{m_{1}}{m_{2}} \frac{1}{\tan \varphi} .
$$

The critical value $\mu_{c}^{+}(\varphi)$ is minimal when $\varphi=\arctan \sqrt{m_{1} / m_{2}}$ giving

$$
\mu_{c \min }=2 \sqrt{\frac{m_{1}}{m_{2}}} .
$$

For $\mu \geqslant \mu_{c \text { min }}$ there exists an interval of $\varphi_{c 1}^{+}<\varphi<\varphi_{c 2}^{+}$for which $A^{+}<0$ with

$$
\varphi_{c 1}^{+}=\arctan \left(\frac{1}{2} \mu+\sqrt{\frac{1}{4} \mu^{2}-\frac{m_{1}}{m_{2}}}\right), \quad \varphi_{c 2}^{+}=\arctan \left(\frac{1}{2} \mu-\sqrt{\frac{1}{4} \mu^{2}-\frac{m_{1}}{m_{2}}}\right) .
$$

With respect to the previous section we have to remark the following lemma.

Lemma 4.1. Consider a mechanical system $\boldsymbol{M}(\boldsymbol{q}) \ddot{\boldsymbol{q}}-\boldsymbol{h}(\boldsymbol{q}, \dot{\boldsymbol{q}})-\boldsymbol{W}_{N}(\boldsymbol{q}) \lambda_{N}-\boldsymbol{W}_{T}(\boldsymbol{q}) \lambda_{T}=\mathbf{0}$ with $n$ degrees of freedom $\boldsymbol{q} \in \mathbb{R}^{n}$ and with a single unilateral contact point, i.e. $\lambda_{N}, \lambda_{T} \in \mathbb{R}$. Let $\boldsymbol{M}(\boldsymbol{q})$ be nonsingular. Let the system be for some nonzero time-interval $t \in U$ in the forward sliding mode, $\lambda_{T}=-\mu \lambda_{N}$, and the detachment LCP be described by $\ddot{g}_{N}=A(\boldsymbol{q}) \lambda_{N}+b(\boldsymbol{q}, \dot{\boldsymbol{q}})$. The motion in the forward slip mode can be described in a set of minimal coordinates $\boldsymbol{a}$ by the reduced system $\mathcal{M}(\boldsymbol{a}) \ddot{\boldsymbol{a}}+\mathcal{H}(\boldsymbol{a}, \dot{\boldsymbol{a}})=\mathbf{0}$, with $\boldsymbol{a} \in \mathbb{R}^{f}, f=n-1$. It holds that $A(\boldsymbol{q})=0$ if and only if $\mathcal{M}(\boldsymbol{a})=\boldsymbol{O}$.

Proof. The proof relies upon a coordinate transformation to minimal coordinates by using the virtual work equations (Glocker, 2001, Section 3.7) after which we substitute the contact force $\lambda_{N}=-b / A$. Let $\boldsymbol{a} \in \mathbb{R}^{f}$ be a set of coordinates such that any position $\boldsymbol{q}$ compatible with the constraint $g_{N}=0$, is uniquely determined by the values of $\boldsymbol{a}$. By such coordinates, the constraints are implicitly fulfilled, i.e. for $\boldsymbol{q}(\boldsymbol{a}, t)$ one has

$$
g_{N}(\boldsymbol{q}(\boldsymbol{a}, t), t) \equiv 0, \quad \forall \boldsymbol{a}, \forall t \in U .
$$

At least locally we may assume the parameterization such that the Jacobian of $\boldsymbol{q}$ with respect to $\boldsymbol{a}$ has full rank $f$. Equation (4.26) also holds for $\boldsymbol{a}^{*}$ near $\boldsymbol{a}$. For $t=t_{c} \in U$ being fixed we expand (4.26) in a Taylor series

$$
g_{N}\left(\boldsymbol{q}\left(\boldsymbol{a}^{*}, t_{c}\right), t_{c}\right) \approx g_{N}\left(\boldsymbol{q}\left(\boldsymbol{a}, t_{c}\right), t_{c}\right)+\frac{\partial g_{N}(\boldsymbol{q})}{\partial \boldsymbol{q}} \frac{\partial \boldsymbol{q}}{\partial \boldsymbol{a}}\left(\boldsymbol{a}^{*}-\boldsymbol{a}\right) .
$$

Taking into account that $\boldsymbol{g}_{N}\left(\boldsymbol{q}\left(\boldsymbol{a}, t_{c}\right), t_{c}\right)=\boldsymbol{g}_{N}\left(\boldsymbol{q}\left(\boldsymbol{a}^{*}, t_{c}\right), t_{c}\right)=0$ by (4.26) we obtain

$$
0=\boldsymbol{W}_{N}^{\mathrm{T}} \boldsymbol{Q} ; \quad \boldsymbol{W}_{N}^{\mathrm{T}}(\boldsymbol{a}, t)=\frac{\partial g_{N}}{\partial \boldsymbol{q}}, \quad \boldsymbol{Q}(\boldsymbol{a}, t)=\frac{\partial \boldsymbol{q}}{\partial \boldsymbol{a}},
$$

with $\boldsymbol{q}=\boldsymbol{q}(\boldsymbol{a}, t)$, because (4.26) holds for every direction $\delta \boldsymbol{a}=\boldsymbol{a}^{*}-\boldsymbol{a} \in \mathbb{R}^{f}$. By differentiation of $\boldsymbol{q}(\boldsymbol{a}, t)$ we obtain

$$
\dot{\boldsymbol{q}}=\boldsymbol{Q} \dot{\boldsymbol{a}}+\tilde{\boldsymbol{\kappa}}, \quad \ddot{\boldsymbol{q}}=Q \ddot{\boldsymbol{a}}+\overline{\boldsymbol{\kappa}},
$$

with $\tilde{\boldsymbol{\kappa}}(\boldsymbol{a}, t)=\partial \boldsymbol{q} / \partial t$ and $\overline{\boldsymbol{\kappa}}(\boldsymbol{a}, \dot{\boldsymbol{a}}, t)=\dot{\boldsymbol{Q}} \dot{\boldsymbol{a}}+\dot{\tilde{\boldsymbol{\kappa}}}$. Evaluation of the virtual work equations (Glocker, 2001) for admissible and nonadmissible virtual displacements using (4.28) and (4.29) gives the two equations:

$$
\begin{aligned}
& \boldsymbol{Q}^{\mathrm{T}} \boldsymbol{M} \boldsymbol{Q} \ddot{\boldsymbol{a}}-\boldsymbol{Q}^{\mathrm{T}}(\boldsymbol{h}-\boldsymbol{M} \overline{\boldsymbol{\kappa}})+\mu \boldsymbol{Q}^{\mathrm{T}} \boldsymbol{W}_{T} \lambda_{N}=\mathbf{0}, \\
& \boldsymbol{W}_{N}^{\mathrm{T}} \boldsymbol{M}^{-1}\left(\boldsymbol{W}_{N}-\mu \boldsymbol{W}_{T}\right) \lambda_{N}+\boldsymbol{W}_{N}^{\mathrm{T}}\left(\boldsymbol{M}^{-1} \boldsymbol{h}-\overline{\boldsymbol{\kappa}}\right)=0 .
\end{aligned}
$$

Note that the term $\mu \boldsymbol{Q}^{\mathrm{T}} \boldsymbol{W}_{T} \lambda_{N}$ does not drop out from the first equation. The second equation of (4.30) can be rewritten as

$$
\ddot{g}_{N}=A \lambda_{N}+b=0,
$$


with

$$
A(\boldsymbol{q})=\boldsymbol{W}_{N}^{\mathrm{T}} \boldsymbol{M}^{-1}\left(\boldsymbol{W}_{N}-\mu \boldsymbol{W}_{T}\right), \quad b(\boldsymbol{q}, \dot{\boldsymbol{q}})=\boldsymbol{W}_{N}^{\mathrm{T}}\left(\boldsymbol{M}^{-1} \boldsymbol{h}-\overline{\boldsymbol{\kappa}}\right)=\boldsymbol{W}_{N}^{\mathrm{T}} \boldsymbol{M}^{-1} \boldsymbol{h}+\overline{\boldsymbol{w}}_{N}
$$

and $\boldsymbol{W}_{N}^{\mathrm{T}} \overline{\boldsymbol{\kappa}}=-\overline{\boldsymbol{w}}_{N}$. The first equation of (4.30) becomes

$$
A \boldsymbol{Q}^{\mathrm{T}} \boldsymbol{M} \boldsymbol{Q} \ddot{\boldsymbol{a}}-A \boldsymbol{Q}^{\mathrm{T}}(\boldsymbol{h}-\boldsymbol{M} \overline{\boldsymbol{\kappa}})-\mu \boldsymbol{Q}^{\mathrm{T}} \boldsymbol{W}_{T} b=\mathbf{0}
$$

which is in the form $\mathcal{M}(\boldsymbol{a}) \ddot{\boldsymbol{a}}+\mathcal{H}(\boldsymbol{a}, \dot{\boldsymbol{a}})=\mathbf{0}$. It follows that $\mathcal{M}(\boldsymbol{a})=\boldsymbol{O}$ if and only if $A(\boldsymbol{q})=0$.

Lemma 4.2. Let $\mu$ and $\boldsymbol{q}$ be such that $A(\boldsymbol{q})=0$, with $\boldsymbol{q}=\boldsymbol{q}(t, \boldsymbol{a})$. It holds that $\mathcal{H}(\boldsymbol{a}, \dot{\boldsymbol{a}})=0$ if and only if $b(\boldsymbol{q}, \dot{\boldsymbol{q}})=0$.

Proof. It follows from Lemma 4.1 that $\mathcal{M}(\boldsymbol{a})=\boldsymbol{O}$ but we cannot immediately state from $\mathcal{M}(\boldsymbol{a}) \ddot{\boldsymbol{a}}=-\mathcal{H}(\boldsymbol{a}, \dot{\boldsymbol{a}})$ that $\mathcal{H}(\boldsymbol{a}, \dot{\boldsymbol{a}})=0$ because the accelerations $\ddot{\boldsymbol{a}}$ are not necessarily bounded. However, if $A(\boldsymbol{q})=0$ then it follows that $b(\boldsymbol{q}, \dot{\boldsymbol{q}})=0$ if and only if $\mathcal{H}(\boldsymbol{a}, \dot{\boldsymbol{a}})=0$.

These lemma's have the following implications for the Frictional Impact Oscillator:

Lemma 4.3. It holds that $A^{+}(\varphi)=0$ if and only if $\mathcal{M}(\varphi)=0$.

Proof. The proof follows immediately from Lemma 4.1 with $\varphi=\boldsymbol{a}$ and $A^{+}(\varphi)=A(\boldsymbol{q}(\boldsymbol{a}))$. For the Frictional Impact Oscillator it holds that $\mathcal{M}(\varphi)=l^{2} N(\varphi) A^{+}(\varphi)$ and $N(\varphi)>0 \forall \varphi$ from which also follows the lemma.

Lemma 4.4. Let $\mu=\mu_{c}^{+}>0$ and be bounded such that $A^{+}(\varphi)=0$. Then it holds that $b(\varphi, \dot{\varphi})=0$ if and only if $\mathcal{D}(\varphi) \dot{\varphi}^{2}+\mathcal{C}(\varphi) \dot{\varphi}+\mathcal{F}(\varphi)=0$.

Proof. The proof follows immediately from Lemma 4.2 with $\mathcal{H}(\boldsymbol{a}, \dot{\boldsymbol{a}})=\mathcal{D}(\varphi) \dot{\varphi}^{2}+\mathcal{C}(\varphi) \dot{\varphi}+\mathcal{F}(\varphi)$. It follows from $\mu=\mu_{c}^{+}>0$ and the restriction that $\mu_{c}^{+}$is bounded that $\sin \varphi \neq 0$. For $\mu=\mu_{c}^{+}$it holds that

$$
b(\varphi, \dot{\varphi})=-\frac{m_{1}}{m_{2}} \frac{\sin \varphi}{N(\varphi) l}\left(\mathcal{D}(\varphi) \dot{\varphi}^{2}+\mathcal{C}(\varphi) \dot{\varphi}+\mathcal{F}(\varphi)\right)
$$

from which follows the lemma.

We can conclude from Lemmas 4.3 and 4.4 that when $\varphi$ is such that $A^{+}=0$, then the slip equation (4.6) is fulfilled only when $b=0$. Note that it must hold in the forward slip mode that $\ddot{g}_{N}=0$ from which follows that $b=0$ when $A^{+}=0$. Let us denote the singular point in the $(\varphi, \dot{\varphi})$-plane for which $A^{+}=0$ and $b=0$ by $P_{c}$. The point $P_{c}$ is located outside the $(\varphi, \dot{\varphi})$-plane shown in Fig. 4.4, at the intersection of the vertical line $A^{+}$and the curve $b=0$. It follows that a solution in the forward slip mode can only reach the line $A^{+}=0$ in the $(\varphi, \dot{\varphi})$-plane through the singular point $P_{c}$. Singular points of this type were studied in detail in (Génot and Brogliato, 1999) for the classical Painlevé example. The contact force $\lambda_{N}$ might become unbounded at $P_{c}$. We assume in the sequel that periodic solutions, if they exist, do not pass the critical point $P_{c}$ (i.e. the critical point is not located on a periodic solution of interest). Indeed, for the hopping phenomenon, which is of interest in the current paper, critical points like $P_{c}$ do not play a role (at least not for the parameter set we study).

The Painlevé paradox can also occur in the backward sliding mode. The friction force $\lambda_{T}$ now works in the opposite direction and the Painlevé paradox can therefore only occur for negative values of $\varphi$. The value $A^{-}$can become negative for sufficiently large values of the friction coefficient $\mu$ and negative values of $\varphi$. The critical value occurs at $\mu=\mu_{c}^{-}$for which $A^{-}=0$. It can easily be verified that $\mu_{c}^{-}=-\mu_{c}^{+}$, being minimal when $\varphi=-\arctan \sqrt{m_{1} / m_{2}}$ giving again the critical value $\mu_{c \text { min }}$ (4.24). For $\mu \geqslant \mu_{c \text { min }}$ there exists an interval of $\varphi_{c 1}^{-}>\varphi>\varphi_{c 2}^{-}$for which $A^{-}<0$ with $\varphi_{c i}^{-}=-\varphi_{c i}^{+}, i=1,2$. However, we will focus in the sequel on the Painlevé paradox in the forward sliding mode because a possible sliding equilibrium is located in the forward sliding mode for $v_{\mathrm{dr}}>0$.

\subsection{Analysis of periodic motion}

The Frictional Impact Oscillator can exhibit periodic motion with slip, stick and flight phases. We will explore how the topology of the periodic solution depends on the regions defined by the lines $A=0$ and $b=0$. We will consider two cases: case 1 with $\mu=0.5<\mu_{c \text { min }}$ and case 2 with $\mu=1>\mu_{c \min }$, where $\mu_{c \min }=0.6325$. The other parameters are $m_{1}=0.1 \mathrm{~kg}$, $m_{2}=1 \mathrm{~kg}, l=1 \mathrm{~m}, k=100 \mathrm{~N} / \mathrm{m}, k_{\varphi}=100 \mathrm{Nm}, c=10 \mathrm{~N} /(\mathrm{ms}), c_{\varphi}=0 \mathrm{Nm} / \mathrm{s}, \varphi_{0}=\pi / 8, v_{\mathrm{dr}}=1 \mathrm{~m} / \mathrm{s}, g=10 \mathrm{Nm} / \mathrm{s}^{2}$. 


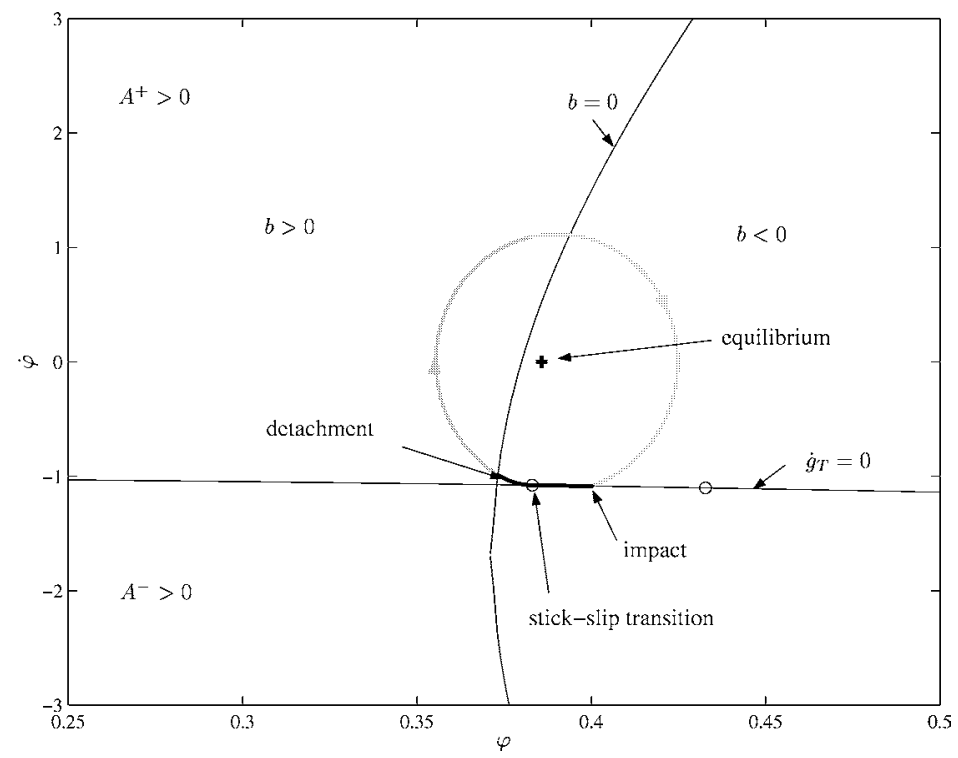

Fig. 4.2. The $(\varphi, \dot{\varphi})$-plane of the Frictional Impact Oscillator, $\mu=0.5$.
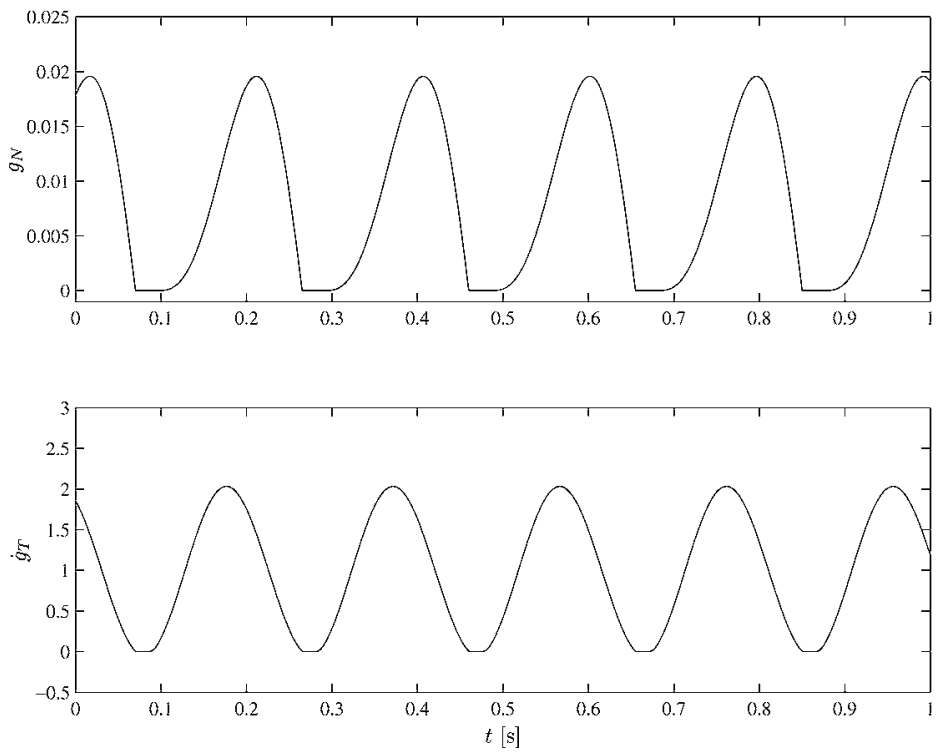

Fig. 4.3. Time history of the Frictional Impact Oscillator, $\mu=0.5$.

Case 1. $\mu=0.5$. Solutions in the forward slip mode exist and are unique because $A>0, \forall \varphi$ for $\mu<\mu_{c \text { min. The lines }}$ $b=0$ (4.21) and $\dot{g}_{T}=0$ are drawn in the plane $(\varphi, \dot{\varphi})$ of Fig. 4.2. The time history of the contact distance $g_{N}$ and contact velocity $\dot{g}_{T}$ are shown in Fig. 4.3. The borders of the friction cone on the line $\dot{g}_{T}$ in Fig. 4.2 are obtained numerically and depicted by a small circle (o). The part of the line $\dot{g}_{T}$ between the two o signs is therefore the stick mode. Note that the friction cone lies, for this parameter set, totally outside the detachment region $A^{+}>0, b>0$. A stable periodic solution is found numerically and consists of the following phases: stick-slip-flight. The dynamics of the flight phase is not only dependent on $(\varphi, \dot{\varphi})$ and is therefore depicted in grey. The stick to slip transition occurs at the border of the friction cone. The body remains some time in forward slipping contact with the belt in the space $A^{+}>0, b<0$ until the line $b=0$ is hit. The sign of $b$ changes at the line $b=0$, giving the LCP solution $\lambda_{N}=0, \ddot{g}_{N}>0$, which means that the body detaches from the belt. The body remains in flight (unconstrained free motion) until an impact occurs $\left(g_{N}=0\right)$. The impact causes the velocities $\dot{\varphi}$ and $\dot{y}$ to jump. However, the jump is so small that the discontinuity cannot be seen at the scale of Figs. 4.2 and 4.3. The impact results 


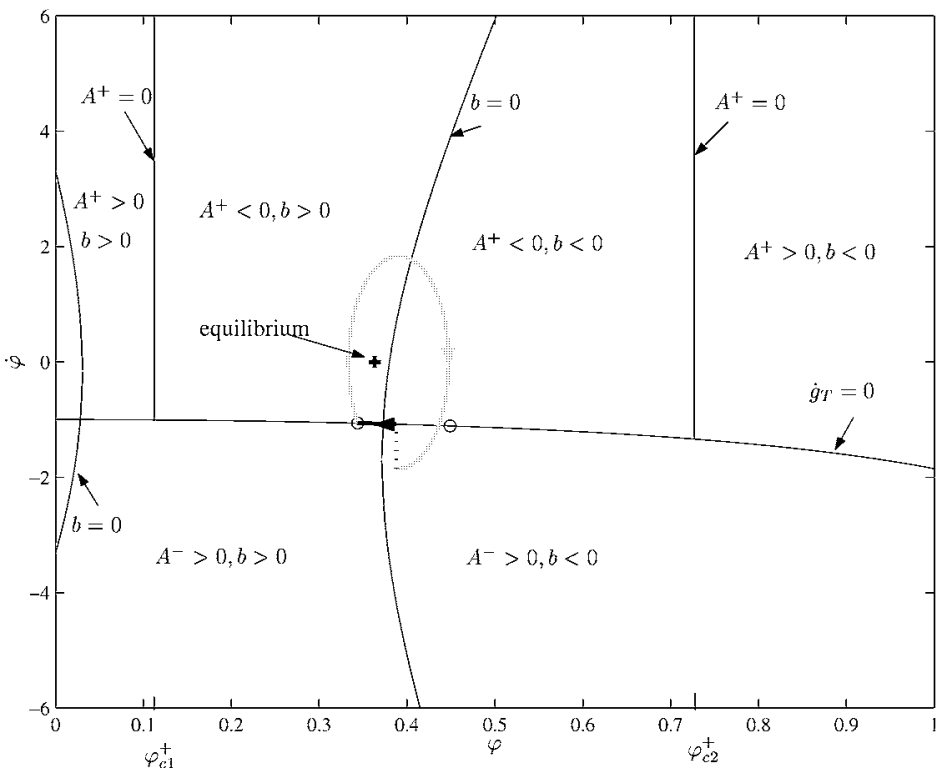

Fig. 4.4. The $(\varphi, \dot{\varphi})$-plane of the Frictional Impact Oscillator, $\mu=1$.
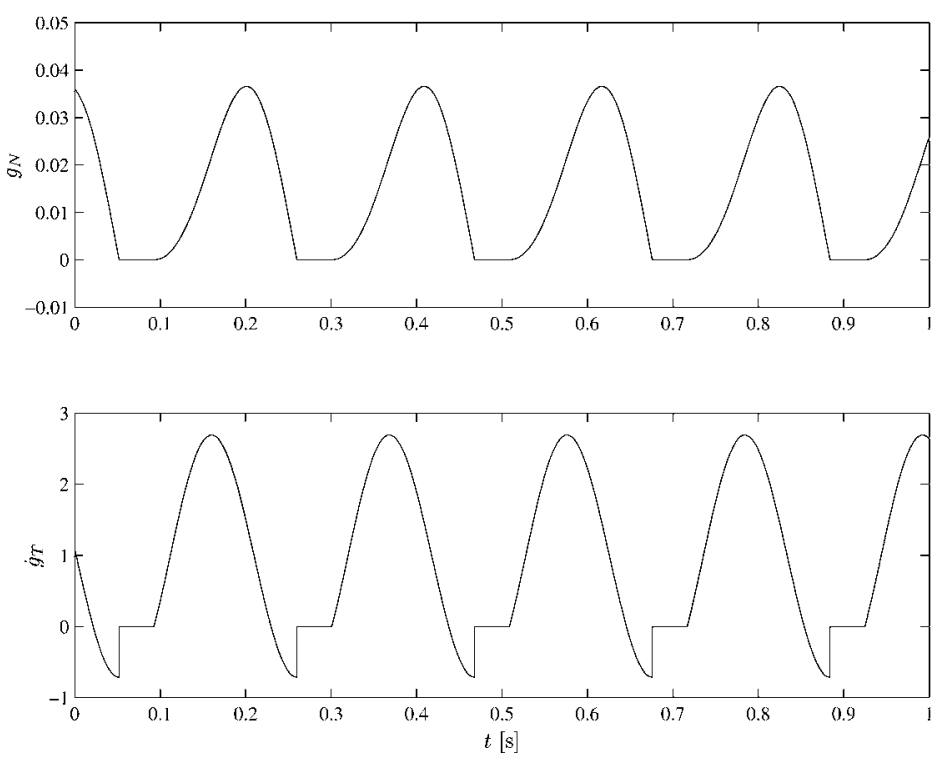

Fig. 4.5. Time history of the Frictional Impact Oscillator, $\mu=1$.

in sticking of the body to the belt. Other coexisting periodic solutions might exist in theory but were not found numerically for this parameter set.

Case 2. $\mu=1$. All four regions of Table 3.1 exist for this parameter set and are depicted in Fig. 4.4. The friction cone is now bordered partly by the region $A^{+}<0, b>0$ (the indeterminate mode of the detachment LCP of the forward slip mode) and is partly bordered by the region $A^{+}<0, b<0$ (the inconsistent mode of the detachment LCP of the forward slip mode). A periodic solution was found which contains a stick phase and a flight phase (depicted in grey). The velocity jump due to the impact is depicted by a dotted line. The impact causes the post-impact state to be in the stick mode. 
A few fundamental questions should be put forward at this point:

(1) Is the solution at the end of the stick phase locally unique? The forward slip mode is at this point undeterminate.

(2) Can an impact cause a post-impact state which is in the forward sliding mode with nonexistence of solution?

These questions are very hard to answer due to the nonlinear terms in the Frictional Impact Oscillator, which make the analysis cumbersome. Instead, it is much more convenient to look at a piecewise linear simplified version of this model, which we call the Simplified Frictional Impact Oscillator. The above questions will be answered for the Simplified Frictional Impact Oscillator. Later, when we look at bifurcation diagrams in Section 6, it will be shown that the Simplified Frictional Impact Oscillator is to some extent a good approximation of the Frictional Impact Oscillator.

\section{The Simplified Frictional Impact Oscillator}

In this section a simplified version of the Frictional Impact Oscillator will be studied. For small values of $\varphi-\varphi_{0} \ll 1$ we can approximate the Frictional Impact Oscillator (Fig. 4.1) with the Simplified Frictional Impact Oscillator depicted in Fig. 5.2. Note that the angle $\gamma$ is constant. Fig. 5.1 might help to understand the relation between the two models. The Frictional Impact Oscillator is pushed over the belt, while the Simplified Bounce Model seems to be dragged over the belt.

\subsection{Model}

The Simplified Frictional Impact Oscillator consists of a mass-spring-damper system $\left(m_{1}, k_{1}, c_{1}\right)$ which stands under a fixed angle $\gamma$ and is attached to a vertically suspended mass-spring-damper system $\left(m_{2}, k_{2}, c_{2}\right)$. The springs are unstressed when $u_{1}=0$ and $u_{2}=0$. The correspondence between the constants of the Frictional Impact Oscillator and the Simplified Frictional Impact Oscillator is $u_{1}=l\left(\varphi-\varphi_{0}\right), u_{2}=y, \gamma=\varphi_{0}, k_{1}=k_{\varphi} / l^{2}, c_{1}=c_{\varphi} / l^{2}, k_{2}=k$ and $c_{2}=c$.

The kinetic and potential energy of the system can be expressed by

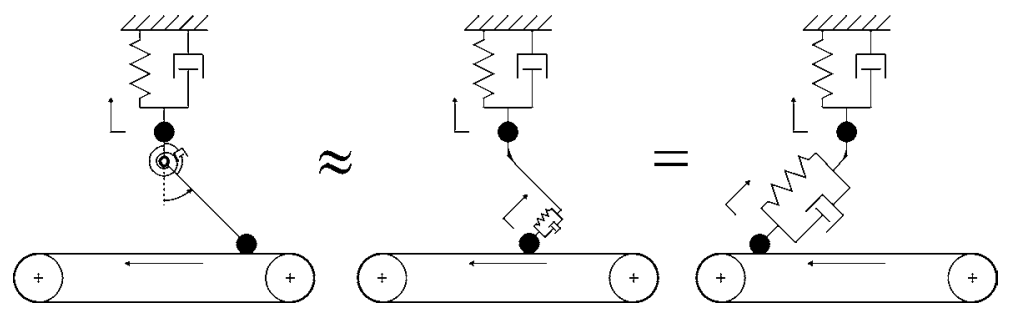

Fig. 5.1. Simplification of the Frictional Impact Oscillator (left) for small rotations of the rod.

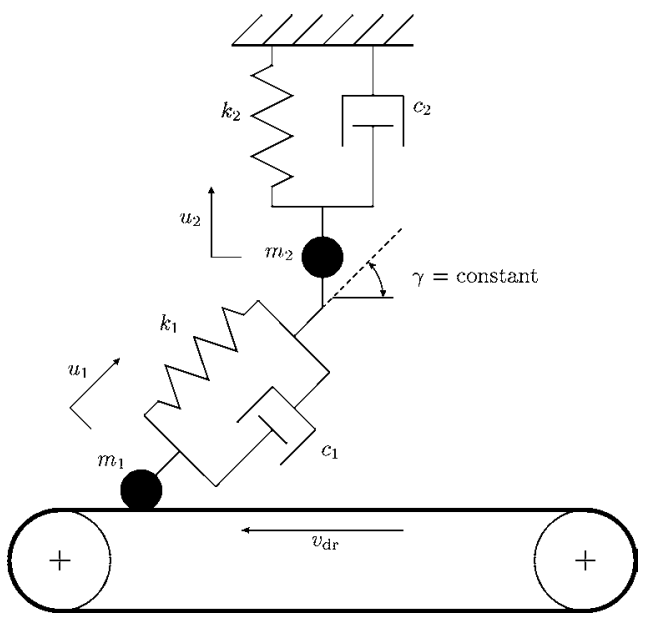

Fig. 5.2. Model of the Simplified Frictional Impact Oscillator. 


$$
\begin{aligned}
T & =\frac{1}{2} m_{1} \dot{u}_{1}^{2}+m_{1} \sin \gamma \dot{u}_{1} \dot{u}_{2}+\frac{1}{2}\left(m_{1}+m_{2}\right) \dot{u}_{2}^{2}, \\
V & =\frac{1}{2} k_{1} u_{1}^{2}+\frac{1}{2} k_{2} u_{2}^{2}+m_{1} g \sin \gamma u_{1}+\left(m_{1}+m_{2}\right) g u_{2} .
\end{aligned}
$$

The system matrices and vectors of the Simplified Frictional Impact Oscillator become

$$
\begin{aligned}
& \boldsymbol{M}=\left[\begin{array}{cc}
m_{1} & m_{1} \sin \gamma \\
m_{1} \sin \gamma & m_{1}+m_{2}
\end{array}\right], \quad \boldsymbol{h}=\left[\begin{array}{c}
-k_{1} u_{1}-c_{1} \dot{u}_{1}-m_{1} g \sin \gamma \\
-k_{2} u_{2}-c_{2} \dot{u}_{2}-\left(m_{1}+m_{2}\right) g
\end{array}\right], \\
& g_{N}=u_{1} \sin \gamma+u_{2}, \quad \dot{g}_{T}=\dot{u}_{1} \cos \gamma+v_{\mathrm{dr}} \\
& \boldsymbol{W}_{N}=\left[\begin{array}{c}
\sin \gamma \\
1
\end{array}\right], \quad \boldsymbol{W}_{T}=\left[\begin{array}{c}
\cos \gamma \\
0
\end{array}\right], \quad \widetilde{\boldsymbol{w}}_{N}=0, \quad \overline{\boldsymbol{w}}_{N}=0, \quad \widetilde{\boldsymbol{w}}_{T}=v_{\mathrm{dr}}, \quad \overline{\boldsymbol{w}}_{T}=0 .
\end{aligned}
$$

\subsection{Forward slip, $\dot{g}_{T}>0$}

The motion of the system in the forward slip mode is constraint with $\lambda_{N} \geqslant 0$ and $\lambda_{T}=-\mu \lambda_{N}$. The equations of motion become

$$
\begin{aligned}
& m_{1} \ddot{u}_{1}+m_{1} \sin \gamma \ddot{u}_{2}+c_{1} \dot{u}_{1}+k_{1} u_{1}=(\sin \gamma-\mu \cos \gamma) \lambda_{N}-m_{1} g \sin \gamma, \\
& m_{1} \sin \gamma \ddot{u}_{1}+\left(m_{1}+m_{2}\right) \ddot{u}_{2}+c_{2} \dot{u}_{2}+k_{2} u_{2}=\lambda_{N}-\left(m_{1}+m_{2}\right) g .
\end{aligned}
$$

Elimination of $\lambda_{N}$ together with the conditions $g_{N}=\dot{g}_{N}=\ddot{g}_{N}=0$ gives a second order differential equation

$$
\mathcal{M}_{\gamma} \ddot{u}_{1}+\mathcal{C}_{\gamma} \dot{u}_{1}+\mathcal{K}_{\gamma} u_{1}=\mathcal{F}_{\gamma},
$$

with

$$
\begin{aligned}
& \mathcal{M}_{\gamma}=m_{1} \cos ^{2} \gamma+m_{2} \sin \gamma(\sin \gamma-\mu \cos \gamma), \quad \mathcal{C}_{\gamma}=c_{1}+c_{2} \sin \gamma(\sin \gamma-\mu \cos \gamma), \\
& \mathcal{K}_{\gamma}=k_{1}+k_{2} \sin \gamma(\sin \gamma-\mu \cos \gamma), \quad \mathcal{F}_{\gamma}=-\left(\left(m_{1}+m_{2}\right) \mu \cos \gamma-m_{2} \sin \gamma\right) g,
\end{aligned}
$$

where the $\mathcal{M}_{\gamma}, \mathcal{C}_{\gamma}, \mathcal{K}_{\gamma}$ and $\mathcal{F}_{\gamma}$ are constants contrary to (4.7). The system has an equilibrium $u_{1 \mathrm{eq}}$ in the forward sliding mode

$$
u_{\text {leq }}=\frac{\mathcal{F}_{\gamma}}{\mathcal{K}_{\gamma}}=\frac{\left(-\left(m_{1}+m_{2}\right) \mu \cos \gamma+m_{2} \sin \gamma\right) g}{\left(k_{1}+k_{2} \sin \gamma(\sin \gamma-\mu \cos \gamma)\right)},
$$

when $\lambda_{N}=-k_{2} u_{1 \text { eq }} \sin \gamma+\left(m_{1}+m_{2}\right) g \geqslant 0$ and $\mathcal{K}_{\gamma} \neq 0$.

The equilibrium is stable if $\mathcal{M}_{\gamma}>0, \mathcal{C}_{\gamma}>0$ and $\mathcal{K}_{\gamma}>0$ or $\mathcal{M}_{\gamma}<0, \mathcal{C}_{\gamma}<0$ and $\mathcal{K}_{\gamma}<0$. The equilibrium undergoes a Hopf bifurcation for $\mathcal{M}_{\gamma}>0, \mathcal{K}_{\gamma}>0$ when the friction coefficient $\mu$ passes the critical value

$$
\mu_{d \gamma}=\tan \gamma+\frac{c_{1}}{c_{2}} \frac{1}{\sin \gamma \cos \gamma}
$$

for which $\mathcal{C}_{\gamma}=0$.

\subsection{Stick mode}

In the stick mode holds $g_{N}=\dot{g}_{N}=\ddot{g}_{N}=\dot{g}_{T}=\ddot{g}_{T}=0$ and $\left|\lambda_{T}\right|<\mu \lambda_{N}$. It follows that $u_{2}=-u_{1} \sin \gamma$ and $\dot{u}_{1}=$ $-v_{\mathrm{dr}} / \cos \gamma$. The normal contact force therefore becomes

$$
\lambda_{N}=c_{2} v_{\mathrm{dr}} \tan \gamma-k_{2} u_{1} \sin \gamma+\left(m_{1}+m_{2}\right) g .
$$

The condition that the contact force must be in the interior of the friction cone, $\left|\lambda_{T}\right|<\mu \lambda_{N}$, results in an interval $u_{\text {stick }}^{-}<u_{1}<$ $u_{\text {stick }}^{+}$with

$$
u_{\text {stick }}^{ \pm}=\frac{c_{1} \frac{v_{\mathrm{dr}}}{\cos \gamma}+(\sin \gamma \pm \mu \cos \gamma)\left(c_{2} v_{\mathrm{dr}} \tan \gamma+\left(m_{1}+m_{2}\right) g\right)-m_{1} g \sin \gamma}{k_{1}+k_{2} \sin \gamma(\sin \gamma \pm \mu \cos \gamma)} .
$$

The solutions to the detachment LCP for the stick mode exist and are unique which follows from

$$
\ddot{g}_{N}=\ddot{u}_{1} \sin \gamma+\ddot{u}_{2}=\frac{1}{m_{1}+m_{2}}\left(\lambda_{N}+c_{2} \dot{u}_{1} \sin \gamma+k_{2} u_{1} \sin \gamma\right)-g .
$$

The stick mode is therefore consistent and determinate. 


\subsection{Painlevé paradox}

Similar to the Frictional Impact Oscillator we analyze the Painlevé paradox of the Simplified Frictional Impact Oscillator in the forward and backward slip mode.

The normal acceleration $\ddot{g}_{N}=\ddot{u}_{1} \sin \gamma+\ddot{u}_{2}$ in the forward and backward slip mode becomes

$$
\ddot{g}_{N}= \begin{cases}A_{\gamma}^{+} \lambda_{N}+b_{\gamma}, & \dot{g}_{T}>0, \\ A_{\gamma}^{-} \lambda_{N}+b_{\gamma}, & \dot{g}_{T}<0,\end{cases}
$$

with

$$
\begin{aligned}
& A_{\gamma}^{+}=\boldsymbol{W}_{N}^{\mathrm{T}} \boldsymbol{M}^{-1}\left(\boldsymbol{W}_{N}-\mu \boldsymbol{W}_{T}\right)=\frac{\cos ^{2} \gamma}{N_{\gamma}}\left(1+\frac{m_{2}}{m_{1}} \tan \gamma(\tan \gamma-\mu)\right) \\
& A_{\gamma}^{-}=\boldsymbol{W}_{N}^{\mathrm{T}} \boldsymbol{M}^{-1}\left(\boldsymbol{W}_{N}+\mu \boldsymbol{W}_{T}\right)=\frac{\cos ^{2} \gamma}{N_{\gamma}}\left(1+\frac{m_{2}}{m_{1}} \tan \gamma(\tan \gamma+\mu)\right), \\
& b_{\gamma}\left(u_{1}, \dot{u}_{1}\right)=\boldsymbol{W}_{N}^{\mathrm{T}} \boldsymbol{M}^{-1} \boldsymbol{h}+\overline{\boldsymbol{w}}_{N}=\frac{\sin \gamma}{N_{\gamma}}\left(\cos ^{2} \gamma\left(c_{2} \dot{u}_{1}+k_{2} u_{1}\right)-\frac{m_{2}}{m_{1}}\left(c_{1} \dot{u}_{1}+k_{1} u_{1}\right)\right)-g
\end{aligned}
$$

and

$$
N_{\gamma}=m_{1} \cos ^{2} \gamma+m_{2} \text {. }
$$

Note that $A_{\gamma}^{ \pm}$is not dependent on $u_{1}$ and $\dot{u}_{1}$. The value $A_{\gamma}^{+}$, belonging to the forward slip mode, can become negative for sufficiently large values of the friction coefficient $\mu$. The critical value occurs at $\mu=\mu_{c \gamma}^{+}$for which $A_{\gamma}^{+}=0$,

$$
\mu_{c \gamma}^{+}=\tan \gamma+\frac{m_{1}}{m_{2}} \frac{1}{\tan \gamma} .
$$

The critical value $\mu_{c \gamma}^{+}$is minimal when $\gamma=\arctan \sqrt{m_{1} / m_{2}}$ giving

$$
\mu_{c \min }=2 \sqrt{\frac{m_{1}}{m_{2}}} .
$$

The Simplified Frictional Impact Oscillator has therefore the same critical friction coefficient for consistency and determinacy of the forward sliding mode as the Frictional Impact Oscillator. The piece-wise linear character of the Simplified Frictional Impact Oscillator allows us however to derive the following important lemma's.

Lemma 5.1. If $\mu=\mu_{c \gamma}^{+}$such that $A_{\gamma}^{+}=0$, then the point $\left(u_{1}, \dot{u}_{1}\right)=\left(u_{\text {stick }}^{-},-v_{\mathrm{dr}} / \cos \gamma\right)$ is located on the line $b_{\gamma}\left(u_{1}, \dot{u}_{1}\right)=0$.

Proof. For $\mu=\mu_{c \gamma}^{+}$(5.18) holds

$$
k_{1}+k_{2} \sin \gamma(\sin \gamma-\mu \cos \gamma)=-\frac{m_{1}}{m_{2}}\left(k_{2} \cos ^{2} \gamma-k_{1} \frac{m_{2}}{m_{1}}\right)
$$

and

$$
\begin{gathered}
c_{1} \frac{v_{\mathrm{dr}}}{\cos \gamma}+(\sin \gamma-\mu \cos \gamma)\left(c_{2} v_{\mathrm{dr}} \tan \gamma+\left(m_{1}+m_{2}\right) g\right)-m_{1} g \sin \gamma \\
\quad=-\frac{v_{\mathrm{dr}}}{\cos \gamma} \frac{m_{1}}{m_{2}}\left(c_{2} \cos ^{2} \gamma-\frac{m_{2}}{m_{1}} c_{1}\right)-\frac{m_{1}}{m_{2}} N_{\gamma} \frac{g}{\sin \gamma} .
\end{gathered}
$$

The border of the friction cone becomes (5.11)

$$
u_{\text {stick }}^{-}=\frac{\frac{v_{\mathrm{dr}}}{\cos \gamma}\left(c_{2} \cos ^{2} \gamma-\frac{m_{2}}{m_{1}} c_{1}\right)+N_{\gamma} \frac{g}{\sin \gamma}}{k_{2} \cos ^{2} \gamma-k_{1} \frac{m_{2}}{m_{1}}} .
$$

Substitution of $u_{1}=u_{\text {stick }}^{-}$and $\dot{u}_{1}=-v_{\mathrm{dr}} / \cos \gamma$ into (5.16) yields $b_{\gamma}\left(u_{1}, \dot{u}_{1}\right)=0$.

Lemma 5.2. If $\mu=\mu_{c \gamma}^{+}$such that $A_{\gamma}^{+}=0$, then the equilibrium point $\left(u_{1}, \dot{u}_{1}\right)=\left(u_{1 \mathrm{eq}}, 0\right)$ is located on the line $b_{\gamma}\left(u_{1}, \dot{u}_{1}\right)=0$. 
Proof. Substitution of $u_{1}=u_{1 \mathrm{eq}}(5.8)$ and $\dot{u}_{1}=0$ into (5.16) together with (5.20) yields $b_{\gamma}\left(u_{1}, \dot{u}_{1}\right)=0$.

The above lemma's show that the equilibrium as well as the left border of the friction cone pass to the left side of the line $b_{\gamma}=0$ when $\mu$ passes the critical value for the Painlevé paradox. The equilibrium and the left border of the friction cone can therefore not be located in, or be bordered by, the inconsistent mode $A_{\gamma}^{+}<0, b_{\gamma}<0$.

\subsection{Analysis of periodic motion}

The Simplified Frictional Impact Oscillator can exhibit periodic motion with slip, stick and flight phases similar to the Frictional Impact Oscillator. We will explore how the topology of the periodic solution depends on the regions defined by the value of $A_{\gamma}^{ \pm}$and the line $b_{\gamma}=0$. We will consider two cases (similar to the two cases of the Frictional Impact Oscillator): case 1 with $\mu=0.5<\mu_{c \gamma}^{+}$and case 2 with $\mu=1>\mu_{c \gamma}^{+}$, where $\mu_{c \gamma}^{+}=0.6556$. The other parameters are $m_{1}=0.1 \mathrm{~kg}, m_{2}=1 \mathrm{~kg}$, $l=1 \mathrm{~m}, k_{1}=100 \mathrm{~N} / \mathrm{m}, k_{2}=100 \mathrm{~N} / \mathrm{m}, c_{1}=0 \mathrm{~N} /(\mathrm{m} \mathrm{s}), c_{2}=10 \mathrm{~N} /(\mathrm{m} \mathrm{s}), v_{\mathrm{dr}}=1 \mathrm{~m} / \mathrm{s}, g=10 \mathrm{~N} \mathrm{~m} / \mathrm{s}^{2}$.

Case 1. $\mu=0.5$. The values of $A_{\gamma}^{ \pm}$are not dependent on $u_{1}$ for the Simplified Frictional Impact Oscillator and are both positive for the parameter set of case 1 . The forward and backward slip modes are therefore always consistent and determinate. The lines $b_{\gamma}=0$ (5.16) and $\dot{g}_{T}=0$ are drawn in the plane $\left(u_{1}, \dot{u}_{1}\right)$ of Fig. 5.3. The time history of the contact distance $g_{N}$ and contact velocity $\dot{g}_{T}$ are shown in Fig. 5.4 for a periodic solution of the system. The borders of the friction cone on the line $\dot{g}_{T}$ in Fig. 5.3 are given by (5.11) and are depicted by a small circle (o). The part of the line $\dot{g}_{T}$ between the two o signs is therefore the stick mode. Note that the friction cone lies, for this parameter set, totally outside the detachment region $b_{\gamma}>0$. A periodic solution is found numerically and consists of the following phases: stick-slip-flight-slip. The numerically obtained Floquet multipliers indicate that the periodic solution is stable, which agrees with its (locally) attracting nature. The stick to slip transition occurs at the border of the friction cone. The body remains some time in forward slipping contact with the belt in the space $b_{\gamma}<0$ until the line $b_{\gamma}=0$ is hit. The sign of $b_{\gamma}$ changes at the line $b_{\gamma}=0$, giving the LCP solution $\lambda_{N}=0, \ddot{g}_{N}>0$, which means that the body detaches from the belt. The body remains in flight (unconstrained free motion) until an impact occurs $\left(g_{N}=0\right)$. The impact causes the velocities $\dot{u}_{1}$ and $\dot{u}_{2}$ to jump. The impact results in slipping contact of the body to the belt. Other coexisting periodic solutions might exist theoretically but were not found numerically.

Case 2. $\mu=1$. The value of $A_{\gamma}^{+}$is negative for case 2 . The friction cone is now partly bordered by the region $b_{\gamma}>0$ (the indeterminate mode of the detachment LCP of the forward slip mode) and partly by the region $b_{\gamma}<0$ (the inconsistent mode of the detachment LCP of the forward slip mode). A periodic solution was found which contains a stick phase. At the end of the stick phase, when the solution is on the border of the friction cone, detachment occurs. The problem is identical to case 2 of the Frictional Impact Oscillator. The detachment LCP has two solutions:

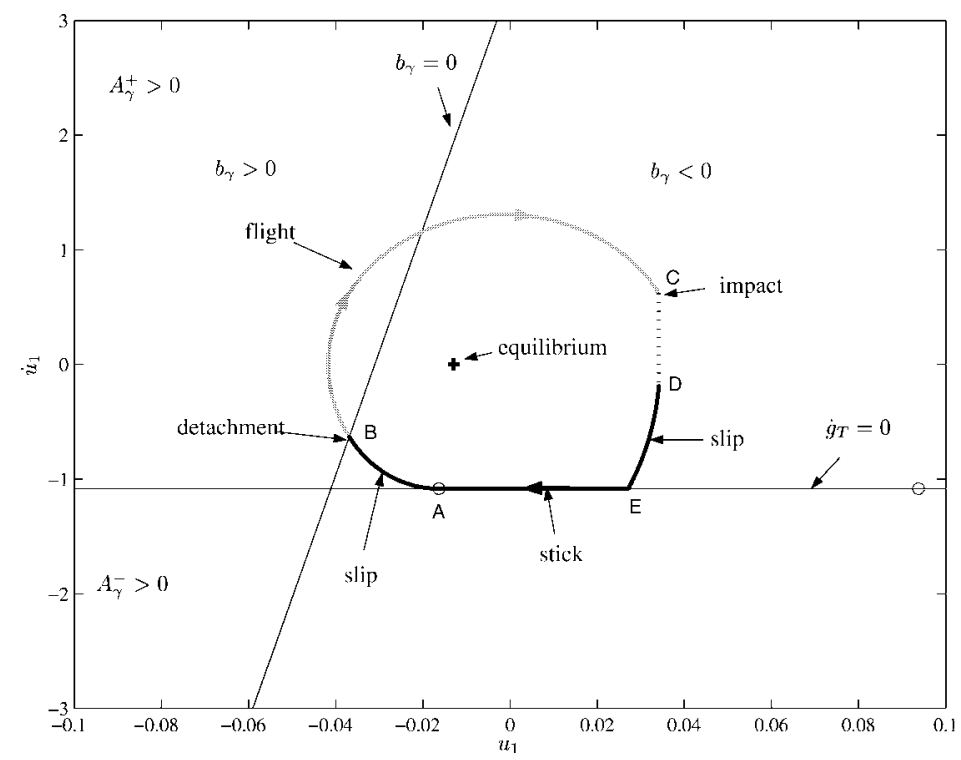

Fig. 5.3. The $\left(u_{1}, \dot{u}_{1}\right)$-plane of the Simplified Frictional Impact Oscillator, $\mu=0.5, A_{\gamma}^{+}=0.5070$. 

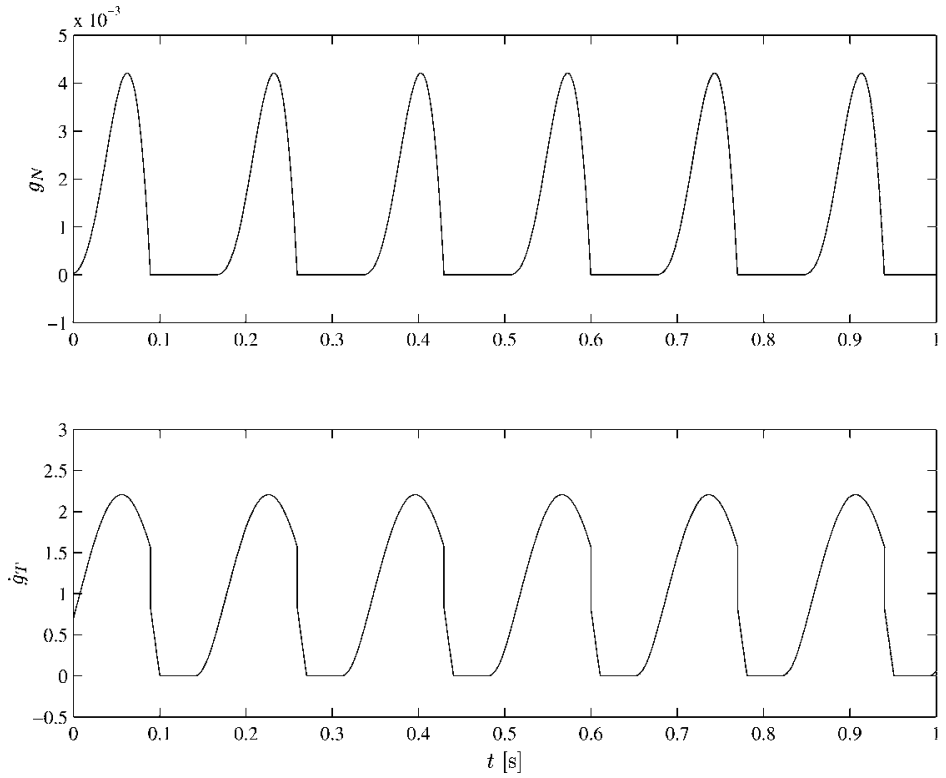

Fig. 5.4. Time history of the Simplified Frictional Impact Oscillator, $\mu=0.5$.

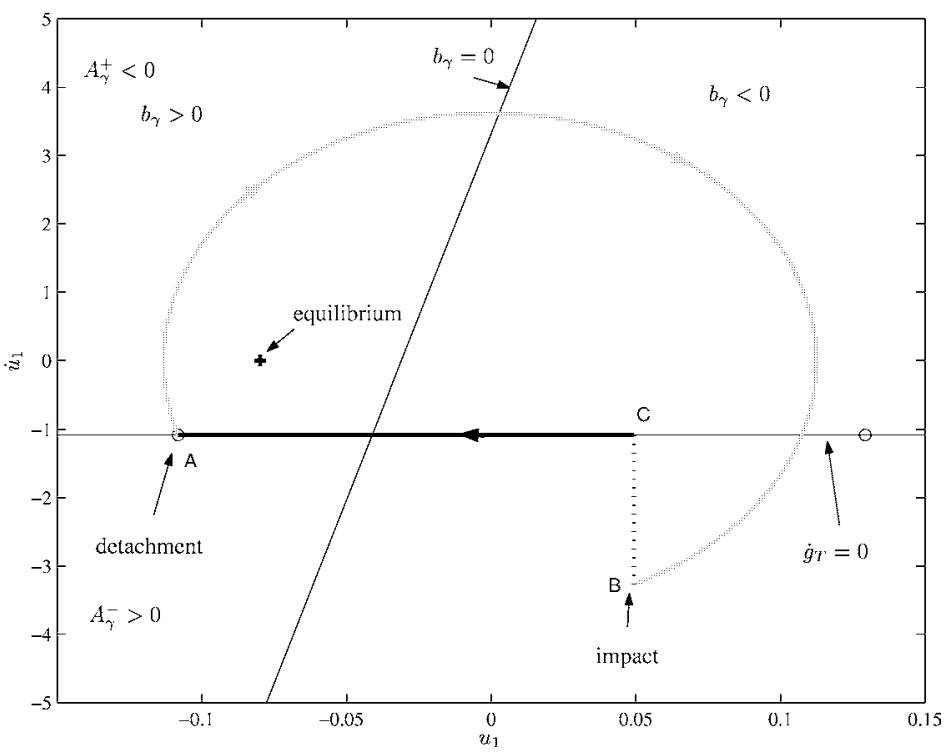

Fig. 5.5. The $\left(u_{1}, \dot{u}_{1}\right)$-plane of the Simplified Frictional Impact Oscillator, $\mu=1, A_{\gamma}^{+}=-1.1218$.

(1) $\lambda_{N}=-b_{\gamma} / A_{\gamma}^{+} \wedge \ddot{g}_{N}=0$, implying forward slip;

(2) $\lambda_{N}=0 \wedge \ddot{g}_{N}=b_{\gamma}$, the body detaches and solution proceeds in a flight phase as the depicted periodic solution does.

The undeterminacy of the detachment LCP at the end of the stick phase for case 2 suggests that the solution $(\boldsymbol{q}(t), \dot{\boldsymbol{q}}(t))$ might be nonunique after this transition. We will have to check rigourously uniqueness at this point. The relationship between uniqueness of solutions of the LCP and uniqueness of solutions $(\boldsymbol{q}(t), \dot{\boldsymbol{q}}(t))$ is not simple in general, see (Brogliato, 1999) $\S 2.2 .3$ and Remark 5.18 therein. Let us therefore consider the following lemma. 

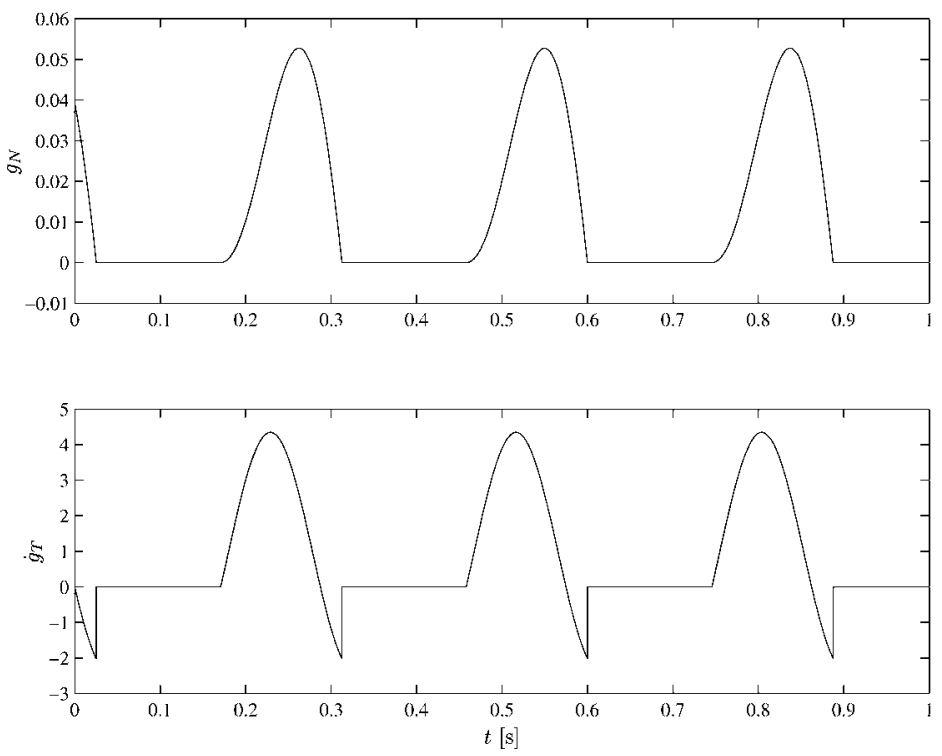

Fig. 5.6. Time history of the Simplified Frictional Impact Oscillator, $\mu=1$.

Lemma 5.3. Let $\mu>\mu_{c \gamma}^{+}, \mathcal{K}_{\gamma}>0$ and

$$
\left(\boldsymbol{q}\left(t_{0}\right), \dot{\boldsymbol{q}}\left(t_{0}\right)\right)=\left[\begin{array}{llll}
u_{\text {stick }}^{-} & -u_{\text {stick }}^{-} \sin \gamma & -\frac{v_{\mathrm{dr}}}{\cos \gamma} & v_{\mathrm{dr}} \tan \gamma
\end{array}\right]^{\mathrm{T}} .
$$

The solution $\boldsymbol{q}(t)$ to the initial value problem of the Simplified Frictional Impact Oscillator with initial condition $\left(\boldsymbol{q}_{0}, \dot{\boldsymbol{q}}_{0}\right)$ at $t=t_{0}$ is locally unique and the solution proceeds in a flight phase $g_{N}>0$.

Proof. It follows from the initial condition $\left(\boldsymbol{q}\left(t_{0}\right), \dot{\boldsymbol{q}}\left(t_{0}\right)\right)$ that $g_{N}\left(t_{0}\right)=\dot{g}_{N}\left(t_{0}\right)=0$. The starting point from $\left(u_{1}\left(t_{0}\right), \dot{u}_{1}\left(t_{0}\right)\right)=$ $\left(u_{\text {stick }}^{-},-v_{\mathrm{dr}} / \cos \gamma\right)$ is at the border of the stick mode $\dot{g}_{T}=0, \lambda_{T}=-\mu \lambda_{N}$. The solution might proceed in the following modes: stick, backward slip, forward slip, flight. Continuation to all of the four modes will be analyzed.

Stick: Assume that a solution $\boldsymbol{q}(t)$ exists on $\left(t_{0}-\varepsilon, t_{0}+\varepsilon\right), \varepsilon>0$. If the solution remains in the stick mode, then $\dot{\boldsymbol{u}}_{1}(t)$ is continuous at $t=t_{0}$. It holds that $u_{1}\left(t_{0}+\varepsilon\right)<u_{1}\left(t_{0}\right), \varepsilon>0$ because $\dot{u}_{1}\left(t_{0}\right)<0$. The solution therefore does not remain in the stick mode $u_{\text {stick }}^{-}<u_{1}<u_{\text {stick }}^{+}$. Continuation in the stick mode is impossible.

Backward slip: The detachment LCP in the backward slip mode is given by $\ddot{g}_{N}=A_{\gamma}^{-} \lambda_{N}+b_{\gamma}$. It holds that $A_{\gamma}^{-}>0$ (5.15). From Lemma 5.1 and the linearity of $b_{\gamma}(5.16)$ in $u_{1}$ and $\dot{u}_{1}$, it follows that $b_{\gamma}\left(t_{0}\right)>0$. Consequently it must hold that $\ddot{g}_{N}\left(t_{0}\right)>0$ and a transition to the flight mode will occur immediately.

Forward slip: The detachment LCP in the forward slip mode is given by $\ddot{g}_{N}=A_{\gamma}^{+} \lambda_{N}+b_{\gamma}$. It holds that $A_{\gamma}^{+}>0$ (5.14) and $b_{\gamma}\left(t_{0}\right)>0$. The detachment LCP for the forward slip mode has two solutions:

(a) $\lambda_{N}=-b_{\gamma} / A_{\gamma}^{+} \wedge \ddot{g}_{N}=0$ which would imply forward slip. If the solution proceeds in the forward slip mode then it holds that

$$
\ddot{g}_{T}\left(t_{0}\right)=\mathcal{M}_{\gamma}^{-1}\left(-\mathcal{C}_{\gamma} \dot{u}_{1}\left(t_{0}\right)-\mathcal{K}_{\gamma} u_{1}\left(t_{0}\right)+\mathcal{F}_{\gamma}\right) \cos \gamma=0 .
$$

Furthermore it holds that $\exists \xi>0, u_{1}\left(t_{0}+\varepsilon\right)=u_{1}\left(t_{0}\right)-\xi$ for an arbitrary small $\varepsilon>0$ because $\dot{u}_{1}\left(t_{0}\right)<0$. It therefore must hold that $\ddot{g}_{T}\left(t_{0}+\varepsilon\right)=\mathcal{M}_{\gamma}^{-1} \mathcal{K}_{\gamma} \xi \cos \gamma<0$ because $\mathcal{M}_{\gamma}<0$ and $\mathcal{K}_{\gamma}>0$. Consequently it holds that $\dot{g}_{T}\left(t_{0}+\varepsilon\right)<0$. The solution will therefore not stay in the forward slip mode but transition to backward slip will occur immediately. From the backward slip mode a transition will occur to the flight mode;

(b) $\lambda_{N}=0 \wedge \ddot{g}_{N}=b_{\gamma}>0$, the solution does not stay in forward slip but transition to the flight mode will occur immediately.

Flight: In the flight mode it holds that $\lambda_{N}=0$. It follows that $\ddot{g}_{N}\left(t_{0}\right)=b_{\gamma}\left(t_{0}\right)>0$. Continuation in the flight mode is possible for a nonzero time interval. Existence of the solution is therefore proven.

It can be concluded that the flight mode is the only possible mode for continuation during a nonzero time interval. The other modes are not possible to enter (stick phase) or switch immediately to the flight mode. The solution will switch to the flight 
mode in a finite number of mode switches and in a zero time interval. The solution is therefore locally unique and proceeds in the flight phase.

Uniqueness of the stick-to-flight transition has been proven. Until now nothing has been said about the existence and uniqueness of the solution after the impact. For the following lemma we will introduce the notation:

$$
\begin{aligned}
& A_{\gamma}(\alpha)=\boldsymbol{W}_{N}^{\mathrm{T}} \boldsymbol{M}^{-1}\left(\boldsymbol{W}_{N}-\alpha \mu \boldsymbol{W}_{T}\right)=\frac{\cos ^{2} \gamma}{N_{\gamma}}\left(1+\frac{m_{2}}{m_{1}} \tan \gamma(\tan \gamma-\alpha \mu)\right), \\
& B_{\gamma}(\alpha)=\boldsymbol{W}_{T}^{\mathrm{T}} \boldsymbol{M}^{-1}\left(\boldsymbol{W}_{N}-\alpha \mu \boldsymbol{W}_{T}\right)=\frac{\cos ^{2} \gamma}{N_{\gamma}}\left(\tan \gamma \frac{m_{2}}{m_{1}}-\alpha \mu\left(1+\frac{m_{2}}{m 1}\right)\right),
\end{aligned}
$$

with $-1 \leqslant \alpha \leqslant 1$. It follows that $A_{\gamma}^{+}=A_{\gamma}(1), A_{\gamma}^{-}=A_{\gamma}(-1)$ and we introduce the abbreviation $B_{\gamma}^{+}=B_{\gamma}(1), B_{\gamma}^{-}=B_{\gamma}(-1)$. Let us prove the following result.

Lemma 5.4. Let $\left(\boldsymbol{q}\left(t_{0}\right), \dot{\boldsymbol{q}}\left(t_{0}^{-}\right)\right)$be an initial condition for which holds $g_{N}\left(t_{0}\right)=0$ and $\dot{g}_{N}\left(t_{0}^{-}\right)<0$ implying an impact at $t=t_{0}$. Let $\mu>\mu_{c \gamma}^{+}$. The post-impact solution $\left(\boldsymbol{q}\left(t_{0}\right), \dot{\boldsymbol{q}}\left(t_{0}^{+}\right)\right)$is locally unique and proceeds in either the backward slip mode, the stick mode or the flight mode.

Proof. The impact equations are

$$
\left[\begin{array}{c}
\dot{g}_{N}\left(t_{0}^{+}\right) \\
\dot{g}_{T}\left(t_{0}^{+}\right)
\end{array}\right]=\left[\begin{array}{ll}
\boldsymbol{W}_{N}^{\mathrm{T}} \boldsymbol{M}^{-1} \boldsymbol{W}_{N} & \boldsymbol{W}_{N}^{\mathrm{T}} \boldsymbol{M}^{-1} \boldsymbol{W}_{T} \\
\boldsymbol{W}_{T}^{\mathrm{T}} \boldsymbol{M}^{-1} \boldsymbol{W}_{N} & \boldsymbol{W}_{T}^{\mathrm{T}} \boldsymbol{M}^{-1} \boldsymbol{W}_{T}
\end{array}\right]\left[\begin{array}{c}
\Lambda_{N} \\
\Lambda_{T}
\end{array}\right]+\left[\begin{array}{c}
\dot{g}_{N}\left(t_{0}^{-}\right) \\
\dot{g}_{T}\left(t_{0}^{-}\right)
\end{array}\right],
$$

with the impulses in normal and tangential direction

$$
\Lambda_{N}=\lim _{t_{0}^{+} \rightarrow t_{0}^{-}} \int_{t_{0}^{-}}^{t_{0}^{+}} \lambda_{N} \mathrm{~d} t, \quad \Lambda_{T}=\lim _{t_{0}^{+} \rightarrow t_{0}^{-}} \int_{t_{0}^{-}}^{t_{0}^{+}} \lambda_{T} \mathrm{~d} t .
$$

For completely inelastic impact it must hold that $\dot{g}_{N}\left(t_{0}^{+}\right)=0$. The post-impact state might be in the forward slip mode, backward slip mode or on the line of zero relative velocity, $\dot{g}_{T}\left(t_{0}^{+}\right)=0$. Continuation in each of the following modes will be analyzed.

Forward slip: We will prove that continuation in the forward sliding mode is impossible by reductio ad absurdum. In the forward slip mode it must hold that $\dot{g}_{T}\left(t_{0}^{+}\right)>0$ and $\Lambda_{T}=-\mu \Lambda_{N}$ with $\Lambda_{N} \geqslant 0$. Substitution into (5.23) gives $A_{\gamma}^{+} \Lambda_{N}+\dot{g}_{N}\left(t_{0}^{-}\right)=0$. However, $A_{\gamma}^{+}<0$ for $\mu>\mu_{c \gamma}^{+}$and $\dot{g}_{N}\left(t_{0}^{-}\right)<0$. Consequently the normal impulse has to be $\Lambda_{N}=-\dot{g}_{N}\left(t_{0}^{-}\right) / A_{\gamma}^{+}<0$ which is contrary to what was assumed before.

Backward slip: In the backward slip mode it must hold that $\dot{g}_{T}\left(t_{0}^{+}\right)<0$ and $\Lambda_{T}=\mu \Lambda_{N}$ with $\Lambda_{N} \geqslant 0$. Substitution into (5.23) gives $A_{\gamma}^{+} \Lambda_{N}+\dot{g}_{N}\left(t_{0}^{-}\right)=0$ from which follows that $\Lambda_{N}=-\dot{g}_{N}\left(t_{0}^{-}\right) / A_{\gamma}^{-}>0$. Furthermore we have $\dot{g}_{T}\left(t_{0}^{+}\right)=B_{\gamma}^{-} \Lambda_{N}+\dot{g}_{T}\left(t_{0}^{-}\right)$. It must hold that $\dot{g}_{T}\left(t_{0}^{+}\right)<0$. Continuation in the backward sliding mode is therefore only possible when $\dot{g}_{T}\left(t_{0}^{-}\right)<\left(B_{\gamma}^{-} / A_{\gamma}^{-}\right) \dot{g}_{N}\left(t_{0}^{-}\right)$.

Zero relative velocity: For a jump to zero relative velocity it must hold that $\exists \alpha, \alpha \in[-1,1]$, for which

$$
\dot{g}_{T}\left(t_{0}^{+}\right)=B_{\gamma}(\alpha) \Lambda_{N}+\dot{g}_{T}\left(t_{0}^{-}\right)=0 \quad \text { and } \quad \Lambda_{N}=-\frac{\dot{g}_{N}\left(t_{0}^{-}\right)}{A_{\gamma}(\alpha)}>0 .
$$

Furthermore, for the stick mode we have the additional condition $u_{\text {stick }}^{-}<u_{1}<u_{\text {stick}}^{+}$. It follows that $A_{\gamma}(\alpha)>0$ to assure $\Lambda_{N}>0$ which gives the condition $\alpha<\mu_{c \gamma}^{+} / \mu$. Substitution of $\Lambda_{N}$ in $\dot{g}_{T}\left(t_{0}^{+}\right)=0$ yields the condition

$$
\frac{\dot{g}_{T}\left(t_{0}^{-}\right)}{\dot{g}_{N}\left(t_{0}^{-}\right)}=\frac{B_{\gamma}(\alpha)}{A_{\gamma}(\alpha)}, \quad \alpha \in\left[-1, \frac{\mu_{c \gamma}^{+}}{\mu}\right) .
$$

The ratio $B_{\gamma}(\alpha) / A_{\gamma}(\alpha)$ attains the values

$$
\frac{B_{\gamma}(\alpha)}{A_{\gamma}(\alpha)} \in\left(-\infty, \frac{B_{\gamma}^{-}}{A_{\gamma}^{-}}\right], \quad \alpha \in\left[-1, \frac{\mu_{c \gamma}^{+}}{\mu}\right) .
$$


A velocity jump to $\dot{g}_{T}\left(t_{0}^{+}\right)=0$ is therefore only possible when $\dot{g}_{T}\left(t_{0}^{-}\right) \geqslant\left(B_{\gamma}^{-} / A_{\gamma}^{-}\right) \dot{g}_{N}\left(t_{0}^{-}\right)$. There are three situations to distinguish:

(a) $u_{\text {stick }}^{-} \leqslant u_{1}\left(t_{0}\right) \leqslant u_{\text {stick }}^{+}$. It is immediate that the post-impact solution will be in the stick mode.

(b) $u_{1}\left(t_{0}\right)>u_{\text {stick}}^{+}$. Continuation in the forward slip mode is impossible as it violates $\lambda_{N}>0$. Continuation in the flight mode is impossible because $b_{\gamma}<0$ for $u_{1}>u_{\text {stick }}^{+}$and $\dot{g}_{T}=0$. Continuation in the backward slip mode is possible if $\ddot{g}_{T}\left(t_{0}^{+}\right)<0$ which is true for $u_{1}>u_{\text {stick }}^{+}$. The solution will proceed in the backward slip mode.

(c) $u_{1}\left(t_{0}\right)<u_{\text {stick }}^{-}$. It follows from Lemma 5.3 that the solution will proceed in the flight mode.

We conclude that the post-impact solution exists and is unique and will proceed either in the backward slip mode, the stick mode or the flight mode, depending on the sign of $\dot{g}_{T}\left(t_{0}^{-}\right) / \dot{g}_{N}\left(t_{0}^{-}\right)-B_{\gamma}^{-} / A_{\gamma}^{-}$and the value of $u_{1}\left(t_{0}\right)$.

\section{Bifurcations}

In the previous sections we analyzed the Frictional Impact Oscillator and in more detail the Simplified Frictional Impact Oscillator. The obtained results will help us to understand the bifurcations which occur when we vary a parameter of these systems. We are interested in bifurcations which occur due to the Painlevé paradox. The friction coefficient $\mu$ is therefore a natural parameter to take as bifurcation parameter.

We will first study a bifurcation diagram of the Simplified Frictional Impact Oscillator and then compare it with a corresponding bifurcation diagram of the Frictional Impact Oscillator. The bifurcation diagram of the Simplified Frictional Impact Oscillator is depicted in Fig. 6.1 with the maximal value of $\left|\dot{u}_{1}\right|$ on the vertical axis and $\mu$ as varying parameter. For small values of $\mu$ we observe that the equilibrium in the forward slip mode (5.8) is stable. The equilibrium loses stability at point $\mathrm{A}$ when $\mu$ is increased to $\mu_{d \gamma}=0.4142$ (5.9) for which $\mathcal{C}_{\gamma}=0$. The equilibrium undergoes a Hopf bifurcation, due to vanishing damping, and a branch of periodic solutions is born (see Fig. 6.2). The equilibrium is located in the interior of a linear region of the piece-wise linear system. An infinite number of marginally stable periodic solutions exist around the equilibrium (i.e. the system locally behaves as a perfect linear system). The marginally stable periodic solution at point D (Fig. 6.2) touches the line $b_{\gamma}=0$. Periodic solutions with a larger norm max $\left|\dot{u}_{1}\right|$ will contain a flight phase and a slip phase. The branch turns due to this nonlinear effect and the periodic solutions on the branch D-B are therefore unstable limit cycles (isolated periodic solutions). The branch turns around at the fold bifurcation point $\mathrm{B}$. The limit cycles between point $\mathrm{B}$ and $\mathrm{C}$ are stable limit cycles which contain slip, stick and flight phases. The limit cycle on the branch B-C for $\mu=0.5$ is depicted in Fig. 5.3. The sign of $\mathcal{M}_{\gamma}$ changes at $\mu=\mu_{c \gamma}^{+}$for which $\mathcal{M}_{\gamma}=0$, i.e., the Painlevé paradox occurs. Points $\mathrm{C}$ and $\mathrm{E}$ are points on the periodic branch and equilibrium branch, respectively, for $\mu=\mu_{c \gamma}^{+}$. The limit cycle on the periodic branch right of point $\mathrm{C}$ for $\mu=1$ is depicted in

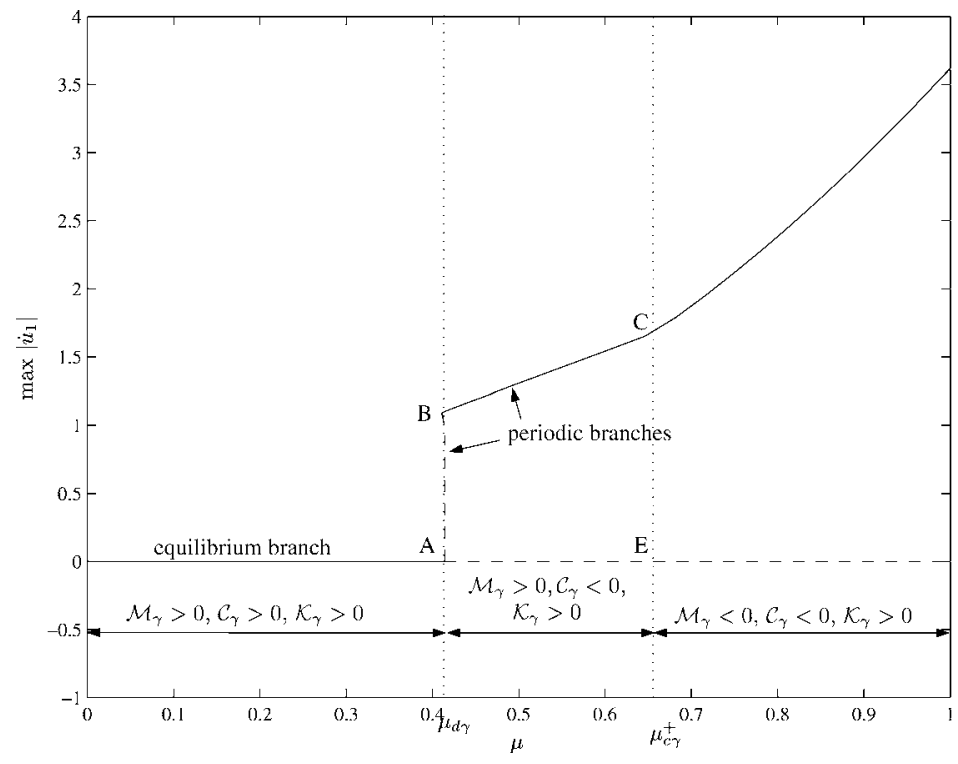

Fig. 6.1. Bifurcation diagram of the Simplified Frictional Impact Oscillator, $c_{1}=0$. 


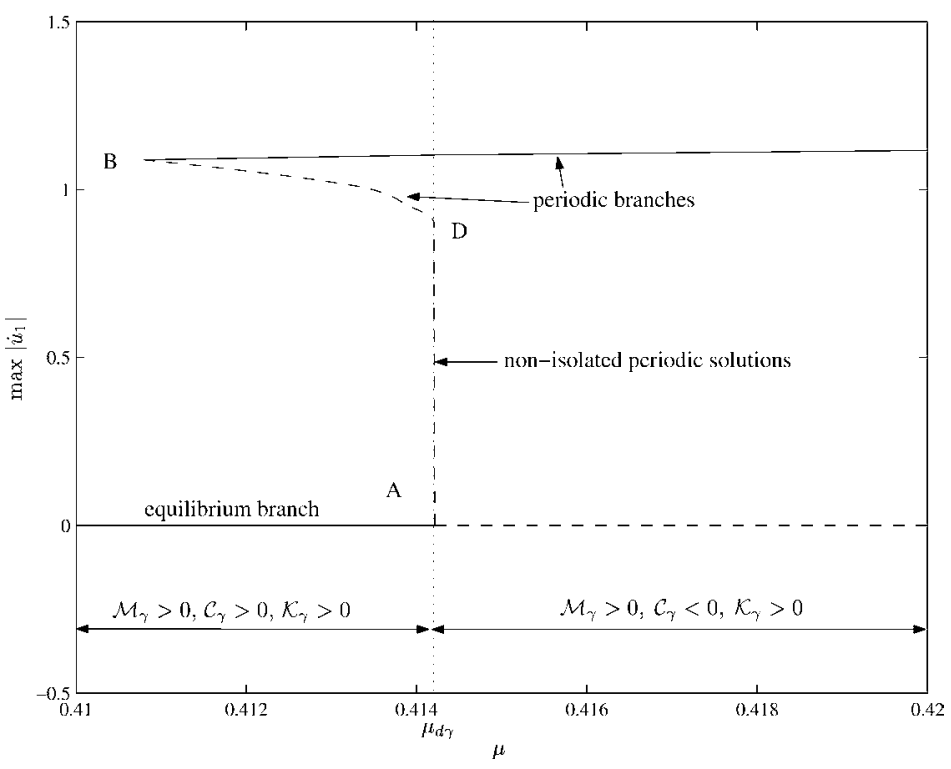

Fig. 6.2. Zoom of Fig. 6.1, $c_{1}=0$.

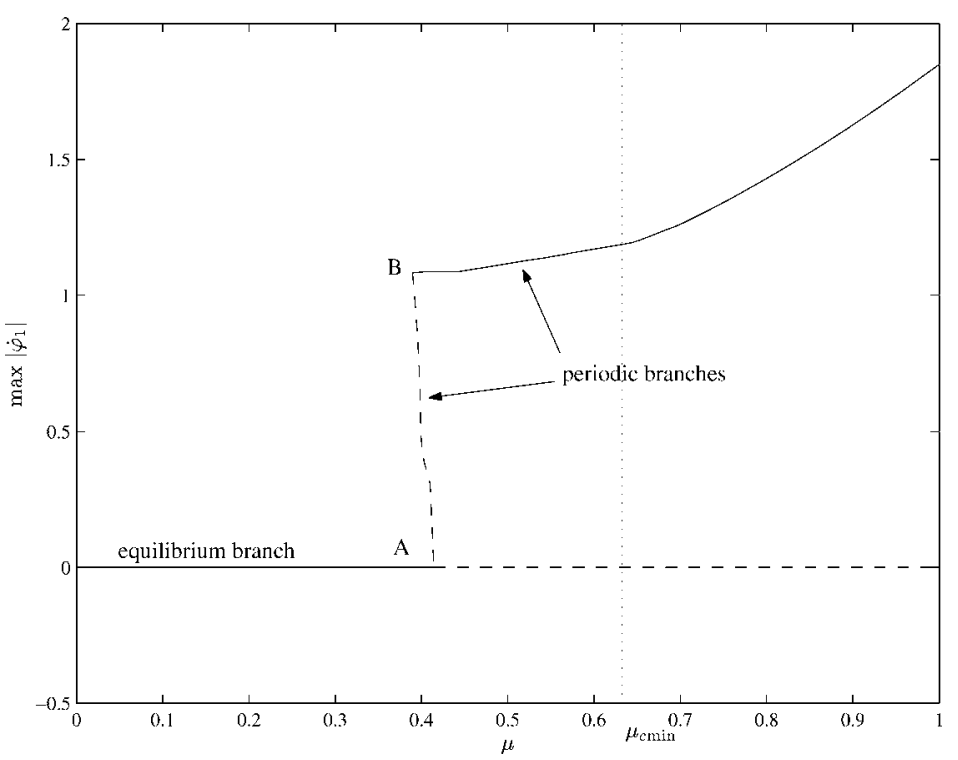

Fig. 6.3. Bifurcation diagram of the Frictional Impact Oscillator (compare with Fig. 6.1).

Fig. 5.5. Lemmas 5.1 and 5.2 hold at point $\mathrm{C}$ and E. Lemma 5.1 states that the border of the friction cone crosses the line $b_{\gamma}=0$ at $\mu=\mu_{c \gamma}^{+}$, which affects the topology of a periodic solution with a stick phase. The periodic solution left of point $\mathrm{C}$ (which looks similar to Fig. 5.3) is therefore topologically different from the periodic solution right of point $\mathrm{C}$ (which looks similar to Fig. 5.5). The branch is nonsmooth at point $C$, but no additional branch is created or destroyed at point $C$. Point $C$ is therefore not a bifurcation point. The topology of the equilibrium changes at point E. According to Lemma 5.2, the equilibrium will be in a space with an indeterminate solution when $\mu>\mu_{c \gamma}^{+}$. The branch left of point $\mathrm{E}$ therefore contains normal equilibria, while the branch right of point $\mathrm{E}$ has equilibria which locally do not have uniqueness of solutions. No additional branch of equilibria or periodic solutions is created at point $\mathrm{E}$, and is therefore not a bifurcation point.

The bifurcation diagram of the Frictional Impact Oscillator is depicted in Fig. 6.3. The stable periodic solution for $\mu=0.5$ corresponds with Fig. 4.2 and for $\mu=1$ with Fig. 4.4. The bifurcation diagram of the Frictional Impact Oscillator looks similar to the bifurcation diagram of the Simplified Frictional Impact Oscillator (Fig. 6.1). However, the Frictional Impact Oscillator 


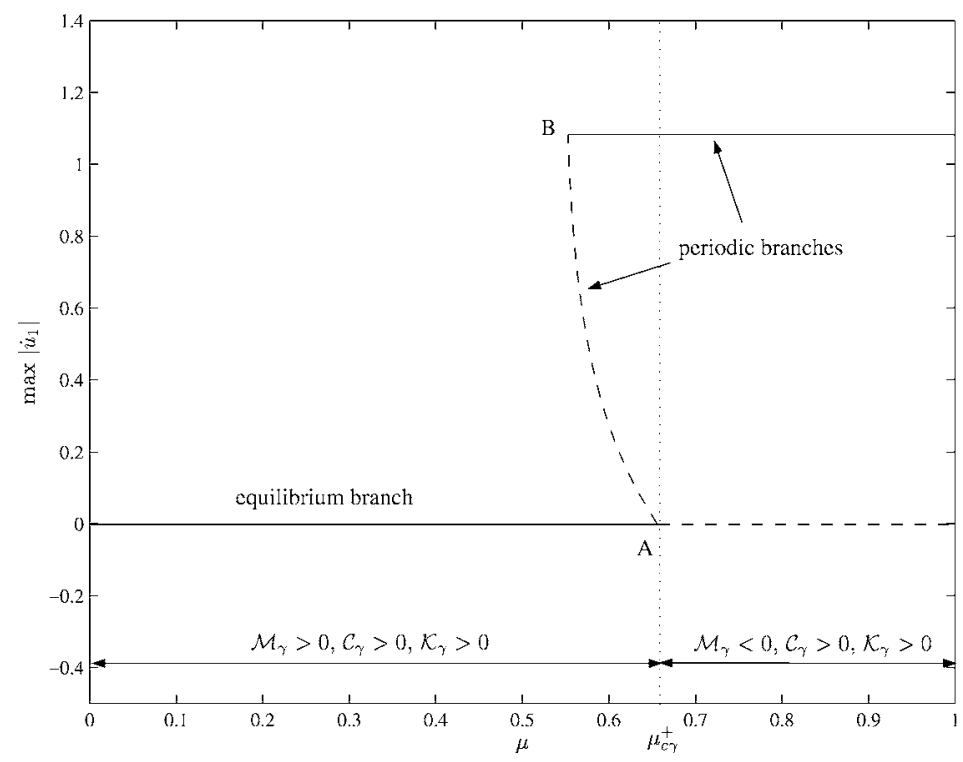

Fig. 6.4. Bifurcation diagram of the Simplified Frictional Impact Oscillator, $c_{1}=10$.

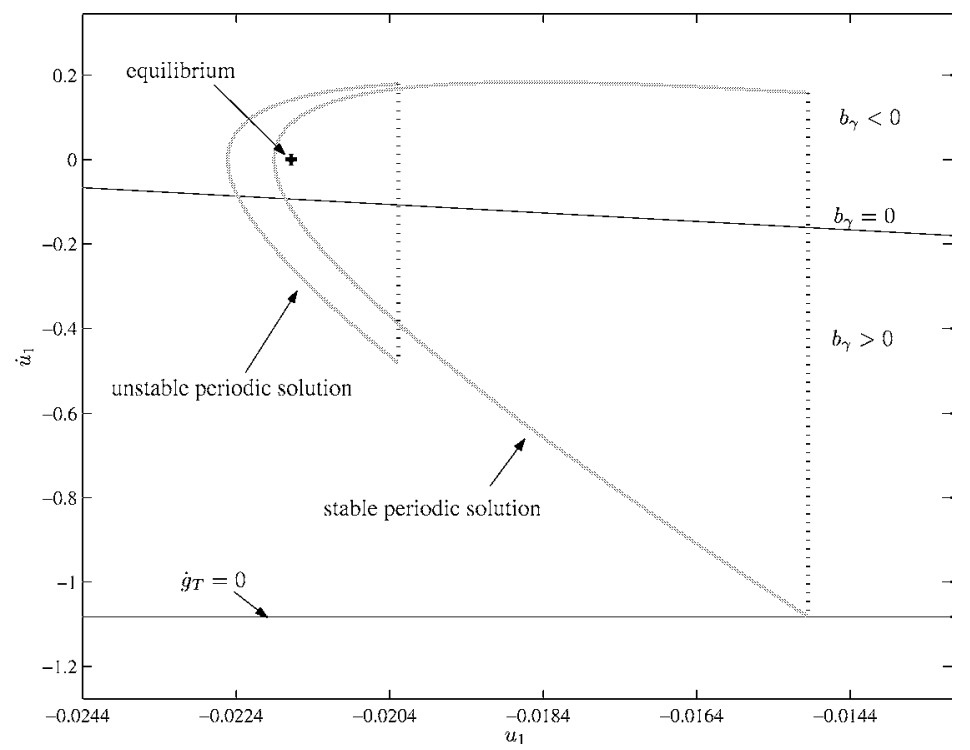

Fig. 6.5. The $\left(u_{1}, \dot{u}_{1}\right)$-plane of the Simplified Frictional Impact Oscillator, $c=10, \mu=0.5773, A_{\gamma}^{+}=0.2553$.

is more complicated than its simplified version and we can give less definite answers. For the Frictional Impact Oscillator we are not equipped with Lemmas 5.1-5.4. We can therefore not say that the equilibrium enters an indeterminate solution when $\mu$ passes $\mu_{c \text { min }}$, although it will happen somewhere in the neighborhood. Likewise, the topology of the stable periodic solution

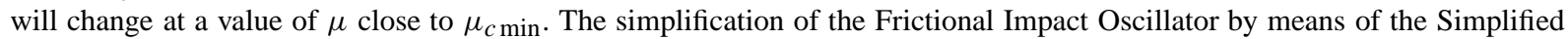
Frictional Impact Oscillator does not only give more insight but also allows us to find exact values of important points in the bifurcation diagram.

The Painlevé paradox played a role in the previous bifurcation diagrams but did not lead to a bifurcation. The Painlevé paradox can however lead to a bifurcation if the parameter set is slightly different. Let us look again at the Simplified Frictional Impact Oscillator. We take the same parameter set as before but now with $c_{1}=10$, leading to $\mu_{d \gamma}>\mu_{c \gamma}^{+}$. The bifurcation diagram is depicted in Fig. 6.4. The equilibrium loses in this case stability when $\mu$ passes the critical value $\mu_{c \gamma}^{+}$(point $\mathrm{A}$ in Fig. 6.4), at which the Painlevé paradox occurs. A branch of unstable periodic solutions originates from point A. Point $\mathrm{A}$ is 
therefore a bifurcation point. The branch of periodic solutions turns around at the fold bifurcation point $\mathrm{B}$. The stable periodic solutions on the branch which starts at point B, are periodic solutions which are characterized by a flight phase followed by an impact to zero relative velocity with immediate detachment (case (c) in the proof of Lemma 5.4). The equilibrium, an unstable periodic solution and a stable periodic solution are coexistent for $0.55<\mu<\mu_{c \gamma}^{+}$. Fig. 6.5 shows the attractors for $\mu=0.5773$. The post-impact velocity of the unstable periodic solution depends on $\mu$, but the post-impact velocity of the stable periodic solution is zero and therefore independent of $\mu$. This explains why the branch of stable periodic solutions is perfectly horizontal.

\section{Conclusions}

In the previous sections we analyzed the Frictional Impact Oscillator and in more detail the Simplified Frictional Impact Oscillator. It was shown that both models have the same critical value of the friction coefficient for the Painlevé paradox. A peculiarity of these models is that the critical friction coefficient depends only on a mass ratio and can therefore be made arbitrary small, i.e.,

$$
\mu_{c \min }=2 \sqrt{\frac{m_{1}}{m_{2}}} .
$$

The Painlevé Paradox can therefore occur at physically realistic values of the friction coefficient. This fact was not apparent from the classical Painlevé example (Fig. 3.1).

As stated in the introduction, many systems exhibit a kind of frictional hopping motion (e.g., a finger or piece of chalk pushed over a table, robot in contact with an obstacle, brake systems). In fact, the Frictional Impact Oscillator (and its simplified version) can be seen as a sort of archetype of these systems, carrying the basic mechanism for the frictional hopping/bouncing phenomenon. What can we conclude from the analysis of the (Simplified) Frictional Impact Oscillator with respect to the existence of hopping/bouncing motion in these type of systems?

- If the mass of the end-effector of the structure, $m_{1}$, is small with respect to the mass of the supporting structure $m_{2}$, then the Painlevé paradox can occur at physically realistic values of the friction coefficient. Take for instance $m_{1} / m_{2}=1 / 36$, which is certainly realistic in robotic applications, then the critical friction coefficient becomes $\mu_{c \text { min }}=1 / 3$.

- Hopping motion can occur when the friction coefficient $\mu$ is large enough such that either

(1) the linear damping terms vanish/become negative $\left(\mu>\mu_{d \gamma}\right.$ for the Simplified Frictional Impact Oscillator);

(2) the Painlevé paradox occurs $\left(\mu>\mu_{c \gamma}\right.$ for the Simplified Frictional Impact Oscillator).

- For many practical applications it might very well be that $\mu_{c \gamma}<\mu_{d \gamma}$ and that the Painlevé paradox is the actual cause of (undesirable) periodic motion. To avoid the hopping phenomenon in these type of systems, one should therefore increase the mass $m_{1}$ of the end-effector.

- The support stiffness $k$ and damping $c$ (or $k_{2}$ and $c_{2}$ of the Simplified Frictional Impact Oscillator) can be looked upon as the PD-action of a position controller for the link with mass $m_{2}$. When the friction is high enough such that $\mu>\tan \varphi$, then the D-action will diminish the dissipation in the system. In fact, the friction causes negative feedback, which can lead to instability of the forward sliding equilibrium.

Bifurcation diagrams of both models were analyzed in Section 6. It was shown that the Painlevé paradox can lead to a bifurcation of branches but not necessarily. In any case the occurrence of the Painlevé paradox will introduce subspaces of the state space in which there is locally no existence or uniqueness. The phase portrait of a dynamical system with the Painlevé paradox is therefore topologically different from the phase portrait of a dynamical system without the Painlevé paradox. If the Painlevé paradox occurs at a critical value of the bifurcation parameter, then a topological transition will occur at this critical point, which might lead to a bifurcation of branches of periodic solutions or equilibria, but not necessarily.

The $(\varphi, \dot{\varphi})$-planes with lines $A=0, b=0$ and $\dot{g}_{T}=0$ (like Figs. 4.4, 4.2 and 5.5, 5.3) proved to be valuable tools when analyzing bifurcations induced by the Painlevé paradox. The lines $A=0, b=0$ and $\dot{g}_{T}=0$ determine the subspaces where detachment, indeterminacy or inconsistency occurs, which greatly affects the topology of periodic solutions and equilibria.

\section{Acknowledgement}

The authors acknowledge the discussions with Ch. Glocker and F. Génot on the Painlevé paradox. 


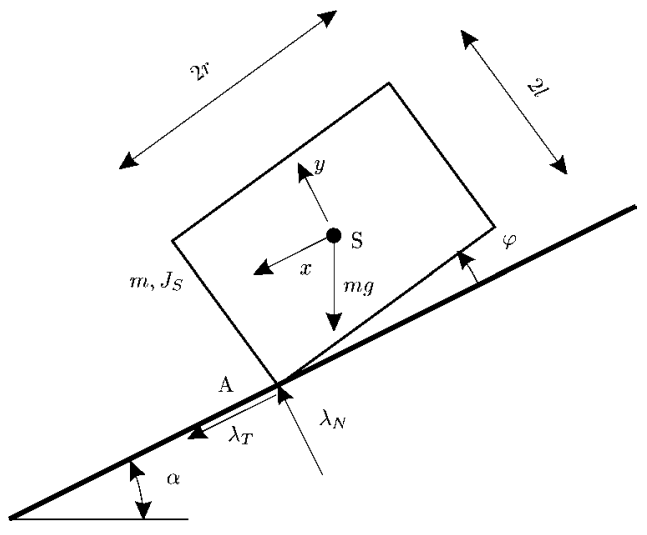

Fig. A.1. The original Painlevé problem.

\section{Appendix A. The original Painlevé problem}

The original example of Painlevé is different from the classical example of Painlevé and is unfortunately forgotten. The original example of Painlevé studied in (Painlevé, 1895b, pp. 114-115) is that of a planar box sliding over an inclined plane. In fact, Painlevé speaks of a three dimensional cylinder with its base in contact with the inclined plane, but this does not make any difference for the planar case. The plane has a slope $\alpha$. The box has the three degrees of freedom $x, y$ and $\varphi$ (its base is parallel to the plane for $\varphi=0$ ). The mass of the box is $m$ and its inertia around the center of mass $S$ is $J_{S}=k^{2} m$, where $k$ is the radius of gyration. Assume that at $t=0$ the box is sliding downward, $\dot{g}_{T}>0$, from which follows that $\lambda_{T}=-\mu \lambda_{N}$. Sliding of the box over the plane is possible when $\mu<r / l$, which can be derived with simple momentum analysis. If $r / l<\mu<r / l+k^{2} / r l$, then the box will turn around the contact point A. If $\mu>r / l+k^{2} /(r l)$, then an inconsistency occurs which is now known as the Painlevé paradox. Assume that the box is slipping in forward direction, $\dot{g}_{T}>0$, and the box begins to rotate, $\varphi=0$. The equations of motion in vertical and angular direction are in this case

$$
m \ddot{y}=-m g \cos \alpha+\lambda_{N}, \quad J_{S} \ddot{\varphi}=-r \lambda_{N}-l \lambda_{T}=(-r+\mu l) \lambda_{N} .
$$

The contact distance $g_{N}$ of point A relative to the slope can be expressed in the coordinates by

$$
g_{N}=y-l \cos \varphi-r \sin \varphi .
$$

The contact acceleration becomes for forward slipping with $\varphi=0$,

$$
\ddot{g}_{N}=\ddot{y}-r \ddot{\varphi}+l \dot{\varphi}^{2} .
$$

Substitution of the equations of motion (A.1) in the contact acceleration (A.3) gives the LCP

$$
\ddot{g}_{N}=\left(\frac{1}{m}-\frac{r}{k^{2} m}(-r+\mu l)\right) \lambda_{N}-g \cos \alpha+l \dot{\varphi}^{2}=A \lambda_{N}+b
$$

with the complementarity condition $0 \leqslant \ddot{g}_{N} \perp \lambda_{N} \geqslant 0$. The solution to the detachment LCP can become nonunique or nonexistent when $\mu$ passes the critical value $\mu_{c}$ with

$$
\mu_{c}=\frac{r}{l}+\frac{k^{2}}{r l} \text {. }
$$

The analysis of Painlevé (1895) ends at this point. Ten years later, in 1905, Painlevé presented a sliding rod problem in his discussion with Lecornu and de Sparre (Lecornu, 1905a, 1905b; de Sparre, 1905; Painlevé, 1905).

If we assume the box to be homogeneous then we obtain $J_{S}=k^{2} m=\frac{1}{3}\left(r^{2}+l^{2}\right) m$. The critical friction coefficient becomes

$$
\mu_{c}=\frac{r}{l}+\frac{1}{3}\left(\frac{r}{l}+\frac{l}{r}\right)
$$

which is minimal for $\frac{r}{T}=\frac{1}{2}$. The minimal friction coefficient is therefore

$$
\mu_{c \min }=\frac{4}{3} \text {, }
$$

which is identical to that of the classical Painleve problem. However, if all the mass is concentrated at $\mathrm{S}$, giving a radius of gyration $k=0$, then $\mu_{c}=r / l$ which can take any positive value. 
Blazejczyk-Okolewska, B., Kapitaniak, T., 1996. Dynamics of impact oscillator with dry friction. Chaos, Solitons \& Fractals 7 (9), $1455-1459$. Brogliato, B., 1999. Nonsmooth Mechanics, 2nd edition. Springer, London.

Dankowicz, H., Nordmark, A.B., 2000. On the origin and bifurcations of stick-slip oscillations. Physica D 136 (3-4), $280-302$.

de Sparre, M., 1905. Sur le frottement de glissement. Comptes Rendu des Séances de l'Academie des Sciences 141, $310-312$.

di Bernardo, M., Feigin, M.I., Hogan, S.J., Homer, M.E., 1999. Local analysis of C-bifurcations in $n$-dimensional piecewise-smooth dynamical systems. Chaos, Solitons \& Fractals 10 (11), 1881-1908.

Foale, S., Bishop, R., 1994. Bifurcations in impacting oscillations. Nonlinear Dynamics 6, 285-299.

Génot, F., 1998. Contributions à la modélisation et à la commande des systèmes avec contraintes unilatérales. PhD thesis, Institut National Polytechnique de Grenoble, France.

Génot, F., Brogliato, B., 1999. New results on Painlevé paradoxes. European Journal of Mechanics A/Solids 18, 653-677.

Galvanetto, U., Knudsen, C., 1997. Event maps in a stick-slip system. Nonlinear Dynamics 13 (2), 99-115.

Glocker, C., 1995. Dynamik von Starrkörpersystemen mit Reibung und Stößen. In: VDI Fortschrittberichte, Vol. 18. VDI-Verlag, Düsseldorf.

Glocker, C., 2000. Scalar force potentials in rigid multibody systems. In: Pfeiffer, F., Glocker, C. (Eds.), Multibody Dynamics with Unilateral Contacts. In: Cism Courses and Lectures, Vol. 421. Springer-Verlag, New York, pp. 69-146.

Glocker, C., 2001. Set-Valued Force Laws, Dynamics of Non-Smooth Systems. In: Lecture Notes in Applied Mechanics, Vol. 1. SpringerVerlag, Berlin.

Guckenheimer, J., Holmes, P., 1983. Nonlinear Oscillations, Dynamical Systems, and Bifurcations of Vector Fields. In: Applied Mathematical Sciences, Vol. 42. Springer-Verlag, New York.

Hensen, R.H.A., van de Molengraft, M.J.G., Leine, R.I., 2002a. Friction induced hunting limit cycles: An event mapping approach. IEEE Transactions on Automatic Control. Submitted.

Hensen, R.H.A., van de Molengraft, M.J.G., Steinbuch, M., 2002b. Friction induced hunting limit cycles: A comparison between the LuGre and switch friction model. Automatica. Submitted.

Ibrahim, R.A., 1994. Friction-induced vibration, chatter, squeal and chaos. Part ii: Dynamics and modeling. ASME Applied Mechanics Reviews 47 (7), 227-253.

Ivanov, A.P., 1996. Bifurcations in impact systems. Chaos, Solitons \& Fractals 7 (10), 1615-1634.

Jellet, J.H., 1872. Treatise on the Theory of Friction. Hodges, Foster and Co.

Lecornu, L., 1905a. Sur la loi de Coulomb. Comptes Rendu des Séances de l'Academie des Sciences 140 (6), $847-848$.

Lecornu, L., 1905b. Sur le frottement de glissement. Comptes Rendu des Séances de l'Academie des Sciences 140 (6), $635-637$.

Leine, R.I., 2000. Bifurcations in discontinuous mechanical systems of Filippov-type. PhD thesis, Eindhoven University of Technology, The Netherlands.

Leine, R.I., Glocker, C., Van Campen, D.H., 2002. Nonlinear dynamics and modelling of some wooden toys with impact and friction. Journal of Vibration and Control. Accepted, 42 pages.

Leine, R.I., Van Campen, D.H., 1999. Fold bifurcations in discontinuous systems. In: Proceedings of DETC'99 ASME Design Engineering Technical Conferences. Las Vegas, CD-ROM, DETC99/VIB-8034.

Leine, R.I., Van Campen, D.H., 2002. Discontinuous bifurcations of periodic solutions. Mathematical Modelling of Nonlinear Systems. Accepted, 20 pages.

Leine, R.I., Van Campen, D.H., Van de Vrande, B.L., 2000. Bifurcations in nonlinear discontinuous systems. Nonlinear Dynamics 23 (2), $105-164$.

Lötstedt, P., 1981. Coulomb friction in two-dimensional rigid-body systems. Zeitschrift für Angewandte Mathematik und Mechanik 61, 605615.

Mason, M.T., Wang, Y., 1988. On the inconsistency of rigid-body frictional planar mechanics. In: Proc. IEEE Int. Conf. Robotics and Automation, CH2555-1, pp. 524-528.

Meijaard, J.P., 1996. A mechanism for the onset of chaos in mechanical systems with motion-limiting stops. Chaos, Solitons \& Fractals 7 (10), 1649-1658.

Moreau, J.J., 1986. Dynamique de systèmes à liaisons unilatérales avec frottement secéventuel; essais numériques. Technical Report Technical Note, 85-1, LMGC, Montpellier, France.

Moreau, J.J., 1988. Unilateral contact and dry friction in finite freedom dynamics. In: Moreau, J.J., Panagiotopoulos, P.D. (Eds.), Nonsmooth Mechanics and Applications. In: International Centre for Mechanical Sciences, Courses and Lectures, Vol. 302. Springer-Verlag, New York, pp. $1-82$.

Nordmark, A.B., 1997. Universal limit mapping in grazing bifurcations. Physical Review E 55 (1), $266-270$.

Painlevé, P., 1895a. Leçon sur le frottement. Hermann, Paris.

Painlevé, P., 1895b. Sur les lois du frottement de glissement. Comptes Rendu des Séances de l'Academie des Sciences 121, 112-115.

Painlevé, P., 1905. Sur les lois du frottement de glissement. Comptes Rendu des Séances de l'Academie des Sciences 141, 401-405 and $546-552$.

Peterka, F., 1996. Bifurcations and transition phenomena in an impact oscillator. Chaos, Solitons \& Fractals 7 (10), $1635-1647$.

Pfeiffer, F., Glocker, C., 1996. Multibody Dynamics with Unilateral Contacts. Wiley, New York.

Popp, K., Hinrichs, N., Oestreich, M., 1995. Dynamical behaviour of a friction oscillator with simultaneous self and external excitation. In: Sādhanā (Ed.), Academy Proceedings in Engineering Sciences. Indian Academy of Sciences, pp. 627-654.

Van de Vrande, B.L., Van Campen, D.H., De Kraker, A., 1999. An approximate analysis of dry-friction-induced stick-slip vibrations by a smoothing procedure. Nonlinear Dynamics 19 (2), 157-169. 
Wiercigroch, M., 1996. On modelling discontinuities in dynamic systems. Machine Vibration 5, 112-119.

Wilms, E.V., Cohen, H., 1981. Planar motion of a rigid body with a friction rotor. Journal of Applied Mechanics 48, $205-206$.

Wilms, E.V., Cohen, H., 1997. The occurence of Painlevé's paradox in the motion of a rotating shaft. Journal of Applied Mechanics 64, 1008-1010.

Yoshitake, Y., Sueoka, A., 2000. Forced self-exited vibration with dry friction. In: Applied Nonlinear Dynamics and Chaos of Mechanical Systems with Discontinuities, Wiercigroch, M., de Kraker, B. (Eds.), World Scientific, pp. 237-257. 